\title{
Phase Behavior of a Block Copolymer in Solvents of Varying Selectivity
}

\author{
Kenneth J . Hanley ${ }^{\dagger}$ and Timothy P. Lodge*, \\ Department of Chemical Engineering \& Materials Science and Department of Chemistry, \\ University of Minnesota, Minneapolis, Minnesota 55455-0431
}

\section{Ching-l Huang}

Graduate Institute of Engineering Technology, National Taiwan University of Science and Technol ogy, Taipei, Taiwan

Received February 22, 2000; Revised Manuscript Received May 17, 2000

\begin{abstract}
The effect of the thermodynamic selectivity of a solvent on the self-assembly of a styreneisoprene (SI) diblock copolymer with block molecular weights of $1.1 \times 10^{4}$ and $2.1 \times 10^{4} \mathrm{~g} / \mathrm{mol}$ is investigated. We explore the phase behavior from the melt state to dilute solution in solvents that are of varying selectivities for the two blocks. Bis(2-ethylhexyl) phthalate (DOP) is a neutral good solvent for SI. Di-n-butyl phthalate (DBP) and diethyl phthalate (DEP) are good solvents for PS but marginal and poor, respectively, for PI. Tetradecane (C14) is utilized as a complementary solvent that is good for PI but poor for PS. Small-angle X-ray scattering (SAXS) and static birefringence are used to locate and identify order-order (OOT) and order-disorder transitions (ODT). Dynamic and static light scattering were used to characterize the dilute sol ution micellar behavior. The neat polymer forms the gyroid $\left(\mathrm{G}_{1}\right)$ morphology at low temperature, an OOT to hexagonal-packed cylinders $\left(C_{1}\right)$ occurs at $185^{\circ} \mathrm{C}$, and the ODT is located at $238{ }^{\circ} \mathrm{C}$. Dilution with DOP decreases the OOT temperature in agreement with the

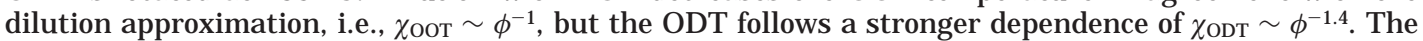
slightly selective solvent DBP stabilizes the ordered state relative to DOP. Rich lyotropic and thermotropic behavior is observed among regions of lamellae $(L)$, inverted gyroid $\left(G_{2}\right)$, inverted hexagonal-packed cylinders $\left(\mathrm{C}_{2}\right)$, and inverted body-centered-cubic spheres $\left(\mathrm{S}_{2}{ }^{\mathrm{bcc}}\right)$. Solutions in DEP display similar morphological behavior, along with significantly increased ODT temperatures. Because of the asymmetric block copolymer composition, the phase behavior in the isoprene-selective solvent C14 is markedly different, as only $G_{1}, C_{1}$, and $S_{1}$ bcc phases are observed. The overall sequence of phases with dilution and/or heating is rationalized on the basis of diagonal trajectories across the phase map (temperature vs composition) for undiluted block copolymers: addition of a neutral solvent corresponds to increasing the temperature and thus a "vertical" trajectory, whereas the partitioning between microdomains of a sel ective solvent amounts to a "horizontal" trajectory to a renormalized block volume composition. However, a variety of novel features are observed in DEP: the formation of face-centered-cubic packed micelles, a reentrant thermotropic ODT, and a large window of $L+C_{2}$ coexistence. The dependence of the principal length scale, $\mathrm{d}^{*}$, on $\phi, \mathrm{T}$, and structure is also considered. The strongly temperature-dependent selectivity produces a crossover in the scaling of $d^{*}$ vs $\phi$ for the lamellar phase: the addition of a selective solvent increases $d^{*}$, but as the solvent becomes neutral, $d^{*}$ decreases. This phenomenon is captured by selfconsistent mean-field calculations.
\end{abstract}

\section{Introduction}

The self-assembly of block copolymers into ordered microstructures continues to be an active area of research. Significant theoretical and experimental effort has been directed at characterizing the phase behavior of the bulk materials, and the factors that govern the selection and location of the various ordered phases in A-B diblock copolymer melts are relatively well understood.1,2 The thermodynamic incompatibility between the $A$ and $B$ segments (dictated by the interaction parameter $\chi$ ) coupled with the low entropy of mixing $\left(\sim N^{-1}\right.$, where $N$ is the total degree of polymerization) controls the degree of segregation between the blocks and is quantified by the product $\chi \mathrm{N}$. At strong segregations, the copolymer will spontaneously organize into periodic A- and B-rich domains. The interfacial curvature and packing symmetry are largely controlled by the copolymer composition, $f$ (block volume fraction). Morphologies include the "classical" phases of lamellae,

$\dagger$ Department of Chemical Engineering \& Materials Science.

‡ Department of Chemistry.

* Author for correspondence. E-mail lodge@chem.umn.edu. hexagonal-packed cylinders, and body-centered-cubic packed spheres, along with the more complex gyroid ( la $\overline{3} d$ symmetry) and metastable perforated lamellae phases. For suitable $\mathrm{N}$ the order-disorder transition (ODT) and order-order transitions (OOT) can be accessed by varying temperature $\left(\chi \sim \mathrm{T}^{-1}\right)$.

Almost all applications of block copolymers involve mixtures, such as blends with a parent homopolymer, low molecular weight "tackifying" resins and plasticizers, or solvent. In block copolymer solutions, a wealth of lyotropic and thermotropic OOTs and ODTs are accessible. Yet, a systematic investigation of the effect of a solvent in transforming the melt phase map for model diblock copolymers has not yet been completed. This situation is additionally complicated since for a given system a solvent that is good for one block can be classified as neutral, slightly selective, or strongly selective, according to whether it is good, near $-\Theta$, or a nonsolvent for the other bl ock. A complete experimental assessment therefore requires expl oring materials with a range of $\mathrm{N}$ and $\mathrm{f}$ to access all initial melt-state morphologies, with differing degrees of segregation. In this work, we focus on a single diblock copolymer and 
characterize its solution phase behavior from the melt state to dilute solution in four solvents of varying selectivities for the two blocks. Furthermore, the sel ectivity has a strong temperature dependence, and consequently small changes in temperature can produce large changes in the solution properties.

The effect of added neutral solvent on the OOT and ODT of diblock copolymers has received considerable attention. ${ }^{3-12}$ In this case, the solvent is distributed nearly uniformly between microdomains, and dilution is therefore analogous to increasing temperature, due to a "screening" of the A-B contacts. Consequently, the melt phase sequence is retained upon dilution, although for asymmetric copolymers exhibiting both an OOT and ODT, a relative reduction of the phase adjacent to the disordered state has been observed. 9,10 Self-consistent mean-field theory is successful in capturing the general features of the experimental phenomena, such as the scaling of the domain spacing with concentration and the slight accumulation of solvent at the microdomain interface. ${ }^{13-19}$ However, the predicted scaling of the transition temperatures with concentration or molecular weight, otherwise known as the "dilution approximation",20 has been found to be incorrect for the ODT ${ }^{7-9}$ but valid for the OOT. ${ }^{9}$ Previous work in selective solvents has tended to focus on dilute solutions where the selectivity drives micellization.1,21 In contrast, studies at nondilute concentrations have been few (but with some exceptions ${ }^{9,22-27}$ ). Aqueous block copolymer solutions systems have received the most attention, particularly in the case of the ethylene oxide-propylene oxide-ethylene oxide (Pluronic) copolymers. ${ }^{21,28-35}$ In this case, water is selective for ethylene oxide, and the polymer solubility decreases with increasing temperature. Phase diagrams have been constructed via theory and experiment and in general show a rich polymorphism with structures that are si milar to those observed in neat block copolymers. However, due to the LCST character of the system and the relatively weak temperature dependence of the solvent selectivity, thermotropic OOTs are less common, and the ODT is difficult to obtain.

Recently, we described the phase behavior of a styrene-isoprene diblock copolymer with 31 vol \% styrene at concentrations near the melt in both the neutral solvent bis(2-ethylhexyl) phthalate (DOP) and the slightly styrene-selective solvent di-n-butyl phthalate (DBP). ${ }^{9}$ DBP is a good solvent for PS but a near- $\Theta$ solvent for $\mathrm{PI}$ at $90{ }^{\circ} \mathrm{C}$. The undiluted material forms the gyroid phase at low temperature, an OOT to hexagonal-packed cylinders occurs at $185^{\circ} \mathrm{C}$, and the ODT is located at $238^{\circ} \mathrm{C}$. In this article we explore the phase behavior for this polymer over the entire concentration range for DBP and also in an increasingly styrene-selective solvent diethyl phthalate (DEP) and an isoprene-selective solvent n-tetradecane (C14) in order to directly investigate the effect of varying the selectivity.

\section{Experimental Section}

Synthesis and Characterization. The synthesis and characterization of the poly(styrene-b-isoprene) diblock copolymer used in this study, designated SI (11-21), has been described in detail. ${ }^{9}$ Styrene and isoprene were polymerized sequentially according to standard living anionic polymerization procedures, yielding a diblock copolymer with numberaverage block molecular weights of $\mathrm{M}_{\mathrm{PS}}=1.1 \times 10^{4} \mathrm{~g} / \mathrm{mol}$ and $M_{\mathrm{PI}}=2.1 \times 10^{4} \mathrm{~g} / \mathrm{mol}$ and $\mathrm{M}_{\mathrm{w}} / \mathrm{M}_{\mathrm{n}}<1.05$, as determined from size-exclusion chromatography (SEC) using both refractive index and multiangle light scattering detectors (MALS). The copolymer polystyrene (PS) volume fraction $\left(f_{P S}\right)$ of 0.31 was calculated from the amount of added monomer and the determined yield ( $>99 \%$ ) assuming densities of 1.05 and 0.913 $\mathrm{g} / \mathrm{mL}$ for PS and polyisoprene (PI), respectively. ${ }^{1 \mathrm{H}} \mathrm{NMR}$ was used to verify the composition and to estimate the mole percent of 1,4-addition of the PI block (94\%).

Sample Preparation. The solvents bis(2-ethylhexyl) phthalate (DOP), di-n-butyl phthalate (DBP), diethyl phthalate (DEP), and n-tetradecane (C14) (Aldrich) were purified by neutralizing with $5 \%$ aqueous sodium bicarbonate, rinsing with distilled water, drying over calcium chloride, and vacuum distilling prior to use. The solutions were prepared gravimetrically using spectrograde methylene chloride as a cosol vent. The cosolvent was stripped off under vacuum at room temperature until a constant weight was achieved. The volume fraction of polymer $(\phi)$ was calculated assuming additivity of volumes and DOP, DBP, DEP, and C14 densities of 0.981, 1.043, 1.118, and $0.763 \mathrm{~g} / \mathrm{mL}$, respectively. The estimated relative uncertainty in $\phi$ is \pm 0.001 for each solution.

Each solution also contained the antioxidant 2,6-di-tertbutyl-4-methylphenol (BHT) ( $0.1 \mathrm{wt} \%$ of polymer) to minimize degradation of the unsaturated PI block. Several control samples were prepared with and without BHT, and as expected, the phase behavior was unaffected. Samples that were subjected to temperatures higher than $175^{\circ} \mathrm{C}$ were routinely checked for degradation using SEC/MALS; no significant degradation was observed.

Small-Angle X-ray Scattering. SAXS experiments were performed on the University of Minnesota beamline. $\mathrm{Cu} \mathrm{K \alpha}$ X-rays $(\lambda=1.54 \AA)$ are generated by a Rigaku RU-200BVH rotating anode, with a $0.2 \times 2 \mathrm{~mm}$ microfocus cathode and Franks mirror optics. Sample temperatures are maintained to within $\pm 0.2{ }^{\circ} \mathrm{C}$ using a water-cooled, electrically heated brass block (temperature range $0-250{ }^{\circ} \mathrm{C}$ ) within a closed sample chamber maintained under a slight positive pressure of helium. Solution samples were sealed within $1.5 \mathrm{~mm}$ quartz capillaries. Two-dimensional diffraction images were collected with a multiwire area detector (HI-STAR, Siemens Analytical $\mathrm{X}$-ray Instruments) at the end of a $2.5 \mathrm{~m}$ flight tube and were corrected for detector response. I mages were azimuthally averaged to the one-dimensional form of intensity (I, arbitrary units) versus scattering wavevector $|\mathbf{q}|=q=4 \pi \lambda^{-1} \sin (\theta / 2)$, where $\theta$ is the scattering angle. The wavevector axis was calibrated using wet duck-foot collagen.

Experiments were performed by first annealing each sample at a set temperature for at least $600 \mathrm{~s}$, followed by a 60-1200 $s \mathrm{X}$-ray exposure. The temperature was then changed in 3 or $5{ }^{\circ} \mathrm{C}$ increments and the procedure repeated. For certain solutions further annealing was performed off-line in a thermostated $\left( \pm 0.1^{\circ} \mathrm{C}\right)$ oil bath to either promotelong-range order or assess structural stability.

Static Birefringence. The apparatus and protocol for the static birefringence (i.e., transmitted depolarized intensity) experiments were performed as follows. Samples were seal ed between two glass disks separated by a $1 \mathrm{~mm}$ aluminum spacer. Vertically polarized light from a $5 \mathrm{~mW}$ HeNelaser was directed through the sample, which was thermostated to within $\pm 0.2^{\circ} \mathrm{C}$ using either an electrically heated (temperature range $40-300^{\circ} \mathrm{C}$ ) or a water-cool ed (temperature range 5-50 $\left.{ }^{\circ} \mathrm{C}\right)$ copper block. The transmitted intensity was directed through a horizontal polarizer and onto a photodiode where the intensity was measured using a voltmeter. Nonzero transmitted intensity is attributed to depolarization due to both form and intrinsic birefringence of the anisotropic PS and $\mathrm{PI}$ domains. 3,36,37 The absence of birefringence is indicative of an isotropic material, i.e., a cubic or disordered phase. The experiment consists of increasing the sample temperature in small increments $\left(1-5{ }^{\circ} \mathrm{C}\right)$ and recording the transmitted intensity after a short equilibration time. The maximum average temperature ramp rate was kept below $1{ }^{\circ} \mathrm{C} / \mathrm{min}$ to promote the observation of equilibrium transition temperatures. 

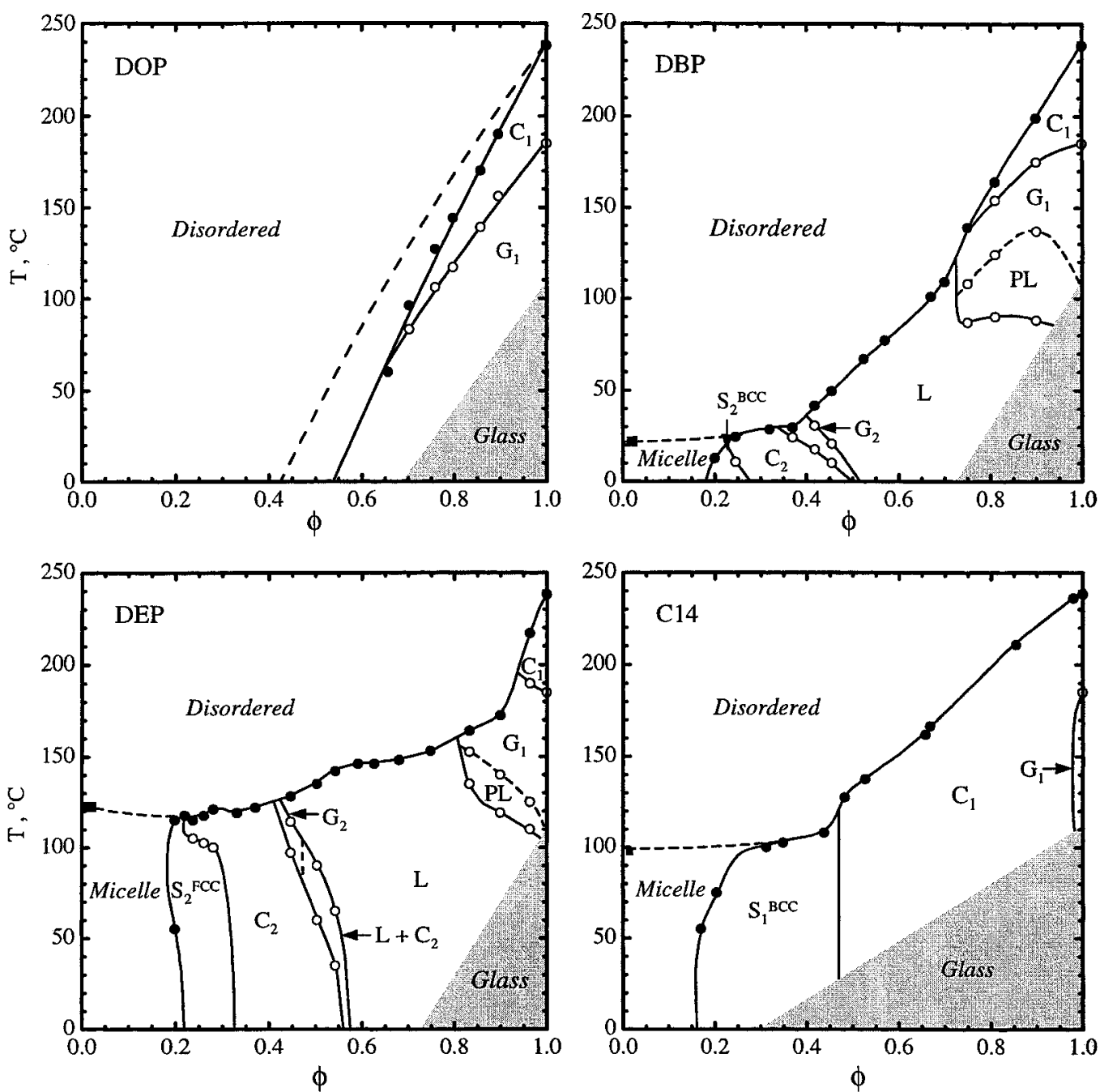

Figure 1. Phase diagram for $\mathrm{SI}(11-21)$ as a function of temperature (T) and polymer volume fraction $(\phi)$ for solutions in DOP, DBP, DEP, and C14. Filled and open circles identify ODTs and OOTs, respectively. The dilute solution critical micelle temperature (cmt) is indicated by a filled square. The ordered phases are denoted by: C, hexagonal-packed cylinders; G, gyroid; PL, perforated lamellae; L, lamellae; S, cubic packed spheres. The subscript 1 identifies the phase as "normal" (PS chains reside in the minor domains) or "inverted" (PS chains located in the major domains). The phase boundaries are drawn as a guide to the eye, except for DOP in which the OOT and ODT phase boundaries (solid lines) show the previously determined scaling of the SI interaction parameter $\left(\chi_{\text {ODT }} \sim \phi^{-1.4}\right.$ and $\left.\chi_{\text {OOT }} \sim \phi^{-1}\right)$; the dashed line corresponds to the "dilution approximation" $\left(\chi_{\text {ODT }} \sim \phi^{-1}\right)$.

Dynamic Light Scattering. Dynamic light scattering (DLS) measurements were made on dilute SI (11-21) solutions in DBP, DEP, and C14 at various concentrations below $\phi=$ 0.02 , to characterize the micellization behavior in the absence of substantial intermicelle interactions. Each sample was passed through an $0.2 \mu \mathrm{m}$ filter prior to use. The DLS spectrometer incorporates a Lexel 95-2 $\mathrm{Ar}^{+}$laser operating at $488 \mathrm{~nm}$, a Brookhaven BI-DS photomultiplier, and a BI-9000 correlator. For each solution, intensity autocorrelation functions $\left(g^{(2)}(t)\right)$ were acquired at various temperatures. At selected temperatures, measurements were made at five angles from $50^{\circ}$ to $130^{\circ}$; at intermediate temperatures only the $90^{\circ}$ intensity correlation function was recorded.

\section{Results}

We first present the phase diagrams of $\mathrm{SI}(11-21)$ in each of the solvents and then discuss the structural characterization of the various phases for each case. In the ensuing Discussion section, we interpret the general features of the phase diagrams in terms of established block copolymer solution principles. Several novel features of the phase behavior are then highlighted and discussed.

Phase Diagrams. The phase diagrams $(\phi, \mathrm{T})$ for $\mathrm{SI}$ (11-21) in DOP, DBP, DEP, and C14 are presented in
Figure 1. The minimum accessible SAXS temperature $\left(0^{\circ} \mathrm{C}\right)$ and maximum acceptable PI exposure temperature to avoid degradation $\left(250^{\circ} \mathrm{C}\right)$ dictated the experimental range. Preliminary structural identification was made using static birefringence, but definitive phase assignments were based on SAXS. The order-disorder and order-order transition temperatures, T ODT (filled circles) and Тоот (open circles), respectively, were determined by combining the results of these techniques. In all solutions examined for this work the two techniques were in excellent agreement (i.e., to within $\pm 1{ }^{\circ} \mathrm{C}$ ). The critical micelle temperatures in dilute solution, cmt (filled squares), were determined using static and dynamic light scattering. The "glass" region approximates where the solvated PS domains are below $\mathrm{T}_{\mathrm{g}}$, thus inhibiting structural equilibration. This window was estimated according to the temperature above which the ordered samples exhibited narrow, intense primary SAXS peaks. The addition of styrene-selective and neutral solvents depressed the glass transition much more rapidly than the isoprene-selective solvent C14, as expected.

In contrast to the neat di block copolymer phase map, where the shorter blocks form the minor domains of a 
given morphology, block copolymer solutions allow the possibility of forming "inverted" phases in which the swollen shorter block plus solvent forms the major domain. We therefore define as "normal" (subscript 1) a structure in which the longer block occupies the major domains and as "inverted" (subscript 2) a phase where the shorter block is located in the major domains. For example, the $\mathrm{C}_{2}$ phase of $\mathrm{SI}(11-21)$ denotes $\mathrm{PI}+$ solvent cylinders in a matrix of PS + solvent. Since SAXS does not readily distinguish normal and inverted structures, the phase assignments were based on the estimated volume fractions of the PS and PI domains.

In studies of phase behavior in general and for block copolymer phase behavior in particular, the thermodynamic/kinetic significance of the observed transition temperatures needs to be addressed. For the phase diagrams presented in Figure 1, the following summary statements apply. Each of the ODTs are reversible, although in more concentrated solutions the ordering on cool ing from the disordered state is much slower than disordering with heating. F urthermore, TODT'S from the two techniques were equivalent within the uncertainty, even though the heating rates were often different. Ther efore, these TODT's may be viewed as approximately equilibrium transition temperatures. TheOOTs arealso reversible, with little or no hysteresis, indicating that they are also equilibrium transition temperatures. The one exception is the perforated-layer (PL) phase, which has been shown to be metastable; 9,38 our results confirm that PL transforms to the bicontinuous gyroid phase of space group Ia $\overline{3} d\left(G_{1}\right)$ with sufficient annealing. The denoted $P L \rightarrow G_{1} T_{\text {OOT }}$ (dashed line in Figure 1 ) should therefore not be viewed as equilibrium transition temperatures but only indicates the transition temperature upon heating a sample at $1^{\circ} \mathrm{C} / \mathrm{min}$. We could not al ways locate the underlying equilibrium lamellar $(\mathrm{L}) \rightarrow \mathrm{G}_{1}$ Tоот, as the time scales can be prohibitive, but upon cooling samples from $G_{1}$, the sample transforms to $L$ slightly below the temperature at which PL first forms upon heating, suggesting that the observed $\mathrm{L} \rightarrow \mathrm{PL}$ transition lies close to the equilibrium $L \rightarrow G_{1} T_{\text {oot }}$.

DOP Phase Diagram. The phase behavior of SI (1121 ) in the neutral solvent DOP was described previously. ${ }^{9}$ The data are replotted in Figure 1 to establish the neutral solvent behavior as a reference in order to reveal the effect of solvent selectivity clearly. Upon dilution with DOP, both the TODT and the $G_{1} \rightarrow C_{1} T_{\text {OOT }}$ decrease rapidly with decreasing $\phi$. The $C_{1}$ temperature window narrows with decreasing concentration until a direct $\mathrm{G}_{1} \rightarrow \mathrm{D}$ ODT occurs at $\phi=0.65$. Increased amounts of solvent $(\phi<0.60)$ produce disordered solutions throughout the experimental temperature range. Consequently, the disordered state in DOP dominates the phase diagram relative to the other solvents.

Figure 1 also displays the concentration scaling of the styrene-isoprene interaction parameter, $\chi$, at the OOT and ODT, as determined previously, ${ }^{9}$ using the following expression (based on the styrene segment volume) determined from extensive results on lamellar-forming SI solutions and melts: ${ }^{7}$

$$
\chi=-0.0228+33 / T
$$

The solid line through the $\mathrm{G}_{1} \rightarrow \mathrm{C}_{1}$ OOTs follows the dilution approximation, i.e., хоот $\sim \phi^{-1}$, whereas the ODTs display the stronger dependence of $\chi_{\mathrm{ODT}} \sim \phi^{-1.4}$, similar to the scaling of $\phi^{-1.6}$ found previously for

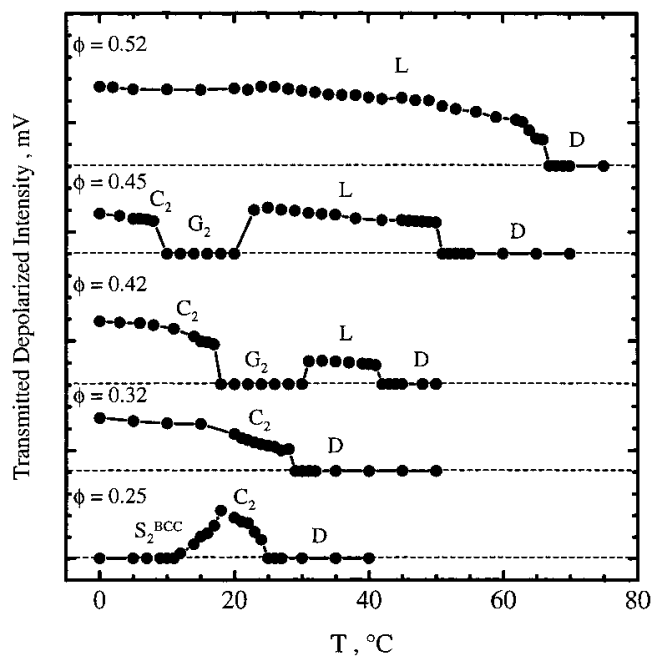

Figure 2. Temperature dependence of the depolarized transmitted intensity for DBP solutions. The dashed lines indicate the baseline after arbitrary vertical shifts.

lamellar diblocks in neutral good solvents. ${ }^{7,8}$ F or comparison, the predicted dilution approximation ${ }^{7}$ scaling of the ODT is shown as the dashed line extending down from the melt TODT. It overestimates TODT by as much as $60{ }^{\circ} \mathrm{C}$ at $\phi=0.55$. The failure of the dilution approximation for the ODT, compared with its success for the OOT, suggests that the disappearance of the $\mathrm{C}_{1}$ phase upon dilution is due to an enhanced stability of the disordered state rather than a slight PS selectivity.

DBP Phase Diagram. DBP was previously shown to behave as a "slightly" styrene-selective solvent; it is a $\Theta$ solvent for PI in the vicinity of $90{ }^{\circ} \mathrm{C}$, but above $100{ }^{\circ} \mathrm{C}$ it becomes effectively neutral.9,22,23 At lower temperatures DBP partitions preferentially into the PS domains. This results in substantial changes to the phase behavior relative to the melt state or DOP as shown in Figure 1.

In concentrated solutions, $\mathrm{T}_{\mathrm{ODT}}$ in DBP is similar to that in DOP, even though the morphology is different; for example, at $\phi=0.70, C_{1} \rightarrow D$ occurs at $100{ }^{\circ} \mathrm{C}$ in DOP, whereas $L \rightarrow D$ occurs at $100^{\circ} \mathrm{C}$ in DBP. As the concentration is decreased bel ow $\phi=0.70$, the difference in TODT increases. By $\phi=0.40$, T ODT becomes almost concentration-independent, signaling a crossover to solvent selectivity-controlled phase behavior. Ultimately, the addition of solvent disrupts the ordered lattice, producing an ODT to a dispersed micellar phase for $\phi \approx 0.2$.

For $0.2<\phi<0.5$, the phase behavior is rich in lyotropic and thermotropic transitions among inverted structures. For example, the $\mathrm{T}$ dependence of the depolarized transmitted intensity is presented for $\phi=$ $0.52,0.45,0.42,0.32$, and 0.25 DBP solutions in Figure 2. At $\phi=0.52$, the sample exhi bits strong birefringence due to the $L$ phase, until at $67^{\circ} \mathrm{C}$ the signal abruptly drops to zero at the ODT. The $\phi=0.45$ solution displays a characteristic "on-off-on-off" temperature dependence that is consistent with the phase sequence $\mathrm{C}_{2} \rightarrow$ $\mathrm{G}_{2} \rightarrow \mathrm{L} \rightarrow \mathrm{D}$. The $\phi=0.42$ solution shows similar behavior with the $\mathrm{C}_{2} \rightarrow \mathrm{G}_{2}$ and $\mathrm{G}_{2} \rightarrow \mathrm{L}$ OOT transitions occurring at higher temperatures, but the $L \rightarrow D$ ODT occurs at a lower temperature. Reducing the concentration to $\phi=0.32$ produces a solution that exhibits strong birefringence at low temperature that persists until approximately $29^{\circ} \mathrm{C}$. This is consistent with the trends established for $\phi=0.45$ and 0.42 in that decreasing the 

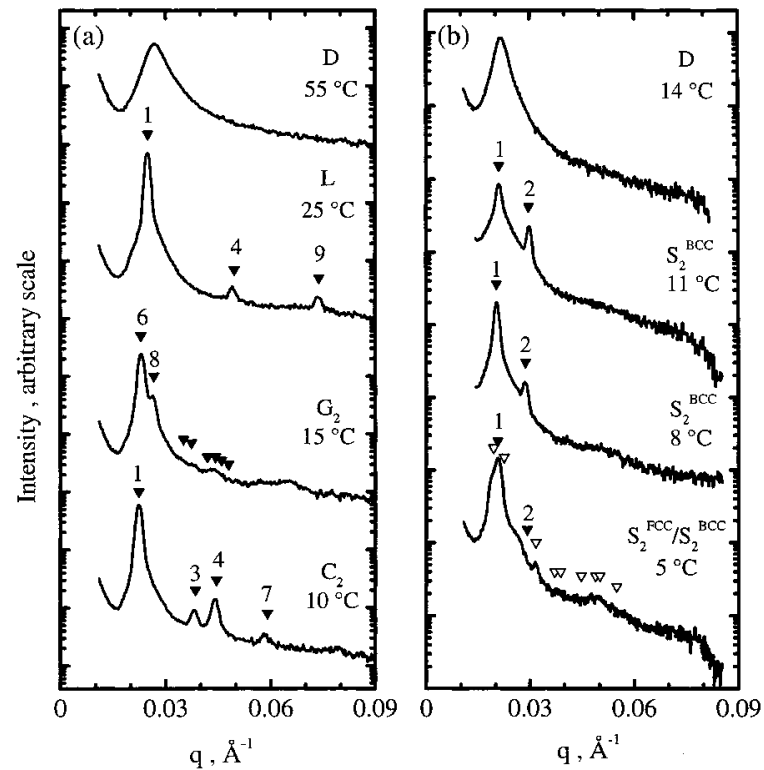

Figure 3. Representative SAXS profiles as a function of temperature for DBP solutions: (a) $\phi=0.45$; (b) $\phi=0.20$. The filled triangles correspond to possible reflections for the stated morphologies at $\mathrm{q} / \mathrm{q}^{*}$ spacings of the indicated squared ratios. The open triangles for the $5^{\circ} \mathrm{C}(\mathrm{b})$ mark $\mathrm{q} / \mathrm{q}^{*}$ spacings of $\sqrt{3}$ : $\sqrt{ } 4: \sqrt{ } 8: \sqrt{ } 11: \sqrt{ } 12: \sqrt{ } 16: \sqrt{ } 19: \sqrt{ } 20: \sqrt{ } 24$ corresponding to an fcc lattice.

concentration leads to an increasing temperature window of $\mathrm{C}_{2}$ yet a decreasing TODT. Ultimately, decreasing the concentration to $\phi=0.25$ leads to the formation of an isotropic phase at low temperature. U pon heating, the structure transforms to $\mathrm{C}_{2}$ at $12{ }^{\circ} \mathrm{C}$ and then disorders at $25^{\circ} \mathrm{C}$, as evident by the abrupt increase and decrease in the transmitted depolarized intensity at the respective transition temperatures.

These phase assignments are all confirmed by SAXS, as illustrated in Figure 3 for $\phi=0.45$ and 0.20 at four selected temperatures. At $10^{\circ} \mathrm{C}$, the $\phi=0.45$ solution (Figure 3a) displays four discernible peaks in the $q$ dependence of the scattered intensity, centered at $\mathrm{q} / \mathrm{q}^{*}$ ratios of $1: \sqrt{ } 3: \sqrt{ } 4: \sqrt{ } 7$, where $q^{*}$ is the principal peak position. This is consistent with the first four allowed reflections for hexagonal-packed cylinders. U pon heating to $15^{\circ} \mathrm{C}$, an OOT is observed as the higher order $\mathrm{C}_{2}$ reflections disappear, and a pronounced shoulder appears on the $q^{*}$ peak. The first two peaks are spaced at ratios of $\sqrt{ } 6: \sqrt{ } 8$, in agreement with the first two allowed reflections of G. ${ }^{39,40}$ Additional allowed reflections are indicated at higher q values, but their assignment is difficult due to the number of possible reflections and the broad peaks. Heating to $25{ }^{\circ} \mathrm{C}$ leads to another thermotropic OOT into $L$, as indicated by the three reflections at peak ratios of $1: 2: 3$, and heating to $55^{\circ} \mathrm{C}$ causes the higher order peaks to disappear and the principal peak broadens considerably. Between 50 and $55{ }^{\circ} \mathrm{C}$, the full width at half-maximum (fwhm) approximately triples, signaling a loss of long-range order in the sample structure. The concomitant loss of birefringence at $51 \pm 1{ }^{\circ} \mathrm{C}$ convincingly identifies the ODT for this solution.

At $8{ }^{\circ} \mathrm{C}$, the $\phi=0.20$ solution (Figure $3 b$ ) displays two strong reflections at $q / q^{*}$ ratios of $1: \sqrt{ } 2$. These results, al ong with the absence of birefringence, and the occurrence of a lyotropic transition from the $\mathrm{C}_{2}$ phase suggest that the structure is probably cubic-packed spheres. The scattering peak ratios conclusively rule out a face-centered lattice ( $\mathrm{fcc}$, space group $\mathrm{F} m \overline{3} \mathrm{~m}$ ), but at least seven reflections would be needed to distinguish between a body-centered (bcc, I I $\overline{3} \mathrm{~m}$ ) and simple cubic (sc) lattice for an unoriented sample. Because of the overwhelming prevalence of bcc structures relative to sc in block copolymer systems, we tentatively conclude the structure is inverted spheres packed on a bcc lattice $\left(\mathrm{S}_{2}{ }^{\mathrm{bcc}}\right)$. Extensive annealing fail ed to produce sufficient long-range order to assign the structure conclusively. Heating the solution produces an ODT as evident by the broadening of the principal peak; $\mathrm{T}_{\mathrm{ODT}}$ for this solution is $12.5 \pm 1.5^{\circ} \mathrm{C}$. Interestingly, cooling the solution to $5^{\circ} \mathrm{C}$ produces a thermoreversible OOT from $\mathrm{S}_{2}$ bcc, as indicated by the broadening of the peak at q* and the emergence of peaks at higher $\mathrm{q}$. For reasons that will be addressed in the Discussion section, we suggest this is due to the formation of an fcc lattice. If the higher $q$ feature at $0.03 \AA^{-1}$ is taken as the $\sqrt{ } 8$ reflection of the fcc lattice, the allowed $\sqrt{ } 3$ and $\sqrt{ } 4$ reflections are in good agreement with the low and high q shoulders of the principal reflection. The remaining portion of the broad q* peak is attributed to scattering from regions of $\mathrm{S}_{2}{ }^{\mathrm{bcc}}$ as evident by the extrapolated temperature dependence of $\mathrm{q}^{*}$ bcc to $5{ }^{\circ} \mathrm{C}$. Because of the numerous allowed fcc reflections at large $q$ and the relatively weak scattering, it is difficult to assign peaks to individual reflections, but there is modest agreement with groups of reflections. Clearly, this is not a wellordered fcc lattice, but we suggest the possibility of either a kinetically hindered $\mathrm{S}_{2}$ bcc $\rightarrow \mathrm{S}_{2}$ fcc transition or coexistence among these structures; prol onged annealing (100 h) failed to change the scattering pattern.

Dilute solution characterization confirms the existence of micelles at low temperature. At $4{ }^{\circ} \mathrm{C}$, the DLS intensity correlation function is dominated by a singleexponential decay. The decay rate is linear in the square of the wavevector, $q$, and thus the corresponding process is diffusive. Application of the Stokes-Einstein relation,

$$
\mathrm{D}=\frac{\mathrm{kT}}{6 \pi \eta_{\mathrm{s}} \mathrm{R}_{\mathrm{h}}}
$$

using the solvent viscosity $\left(\eta_{\mathrm{s}}\right)$ of $49 \mathrm{cP}$ yields a hydrodynamic radius, $R_{h}$, of $173 \pm 5 \AA$. This is clearly far in excess of the size of a single coil for this molecular weight. Figure $4 a$ presents the results obtained from an equivalent analysis for the temperature dependence of $R_{h}$ in each of the selective solvents. For DBP, $R_{h}$ remains approximately constant with increasing temperature before abruptly decreasing from $153 \pm 4 \AA$ at $17{ }^{\circ} \mathrm{C}$ to approximately $62 \pm 5 \AA$ at $22^{\circ} \mathrm{C}$, a value more appropriate for a single coil. Consequently, one may conclude that the increase in temperature is sufficient to overcome the enthalpic drive toward micellization. This conclusion is supported by the static scattered light intensity at fixed angle for dilute solutions of SI (11-21) shown in Figure 4b. The strong scattering observed for the DBP solutions rapidly decreases with increasing temperature with a sharp break at $22{ }^{\circ} \mathrm{C}$, after which the scattered intensity is weak and nearly temperatureindependent. The critical micelle temperature $(\mathrm{cmt})$ is assigned as $22 \pm 1{ }^{\circ} \mathrm{C}$ for this system; it is a direct consequence of the temperature-dependent solvent quality toward the isoprene block. Finally, it is noteworthy that the cmt corresponds well with TODT for solutions near $\phi=0.2$, suggesting that both transitions reflect similar origins. 


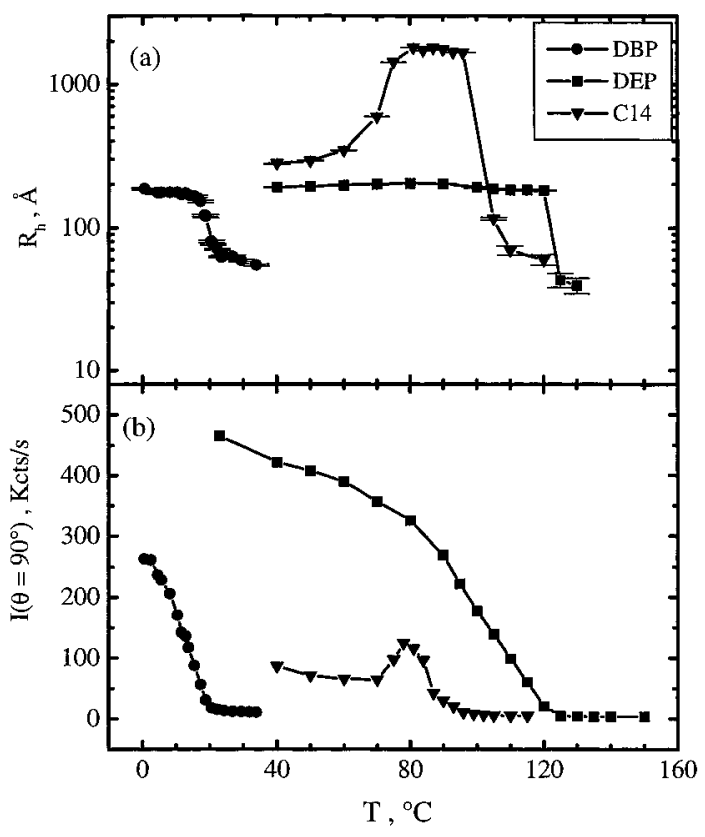

Figure 4. Temperature dependence of the hydrodynamic radius, $R_{h}(a)$, and scattered intensity (b) for solutions of SI(11-21) at $\phi=0.01$ for DBP and $\phi=0.015$ for DEP and C14.

DEP Phase Diagram. Decreasing the alkyl chain length of dialkyl phthalates increases the selectivity toward styrene, and therefore DEP should be more styrene-selective than DBP. The phase diagram of SI(11-21) in DEP is presented in Figure 1. Qualitatively, the results are similar to SI(11-21) in DBP, but there are several significant differences. Most notably, the effect of the greater selectivity is demonstrated by the significant increase in TODT relative to both DOP and DBP for concentrations less than $\phi=0.8$. At higher concentrations, TODT decreases as a strong function of $\phi$, with values that are similar to those in DOP and DBP, thus indicating the effectively neutral behavior of the solvent at high temperatures. At lower $\phi$, the concentration dependence of TODT is much weaker than for DBP; T ODT decreases by less than $50{ }^{\circ} \mathrm{C}$ from $\phi=$ 0.9 to 0.2 . Consequently, TODT in DEP is as much as $100{ }^{\circ} \mathrm{C}$ higher than in DBP.

As anticipated, an increase in the cmt in dilute solution accompanies the increased stability of the ordered phases at higher concentrations. Evidence of the $\mathrm{cmt}$ is again twofold: the scattered intensity rapidly decreases to near zero at $122{ }^{\circ} \mathrm{C}$ (Figure 4b), and the temperature dependence of the hydrodynamic radius (Figure 4a) displays a similar abrupt decrease in size from roughly 180 to $45 \AA$ at $122^{\circ} \mathrm{C}$. There is little effect of solvent selectivity on the micelle size, as $R_{h}$ is approximately the same for micelles in DBP and DEP. Furthermore, in DEP $R_{h}$ is nearly temperature-independent bel ow the cmt. This has also been observed in similar organic micellar solution systems upon varying the solvent selectivity near the $\mathrm{cmt}^{21,41}$ However, it was also found that the aggregation number (and thus radius) increases with increasing selectivity for the coronal block. It is possible that $R_{h}$ remains independent of selectivity due to competing changes in the micelle structure; the increase in aggregation number with decreasing temperature (indicated by the progressive increase in scattered intensity in Figure 4b) may be offset by a decrease in the core radius as solvent is

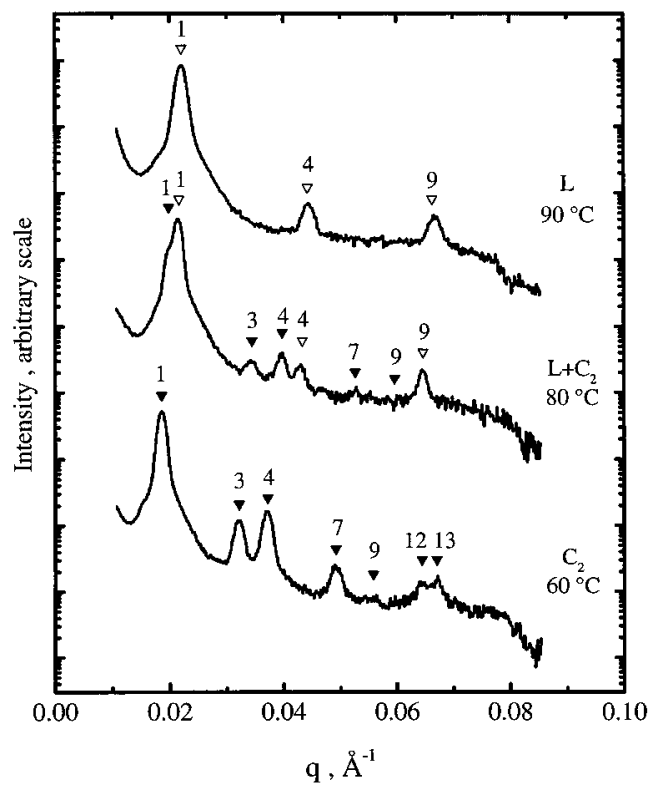

Figure 5. Representative SAXS profiles as a function of temperature for a $\phi=0.50 \mathrm{DE} P$ solution. The filled and open triangles correspond to possible reflections for the $C_{2}$ and $L$ morphologies, respectively, at $q / q^{*}$ spacings of the indicated squared ratios.

excluded from the core and the micelle becomes more dense.

I ncreasing the solvent selectivity does produce several significant changes in the topology of the phase diagram. These features are identified here, but a discussion of the possible underlying reasons is deferred to the Discussion section. First, a two-phase window of $L$ coexisting with $C_{2}$ is found near $\phi=0.55$ in between the respective single-phase windows. The SAXS signature of the thermotropic transitions between these structures is shown in Figure 5 for the $\phi=0.50$ solution. At $60^{\circ} \mathrm{C} \mathrm{C} C_{2}$ forms with significant long-range order, as evident by the seven clear reflections at $q / q^{*}$ ratios of $1: \sqrt{ } 3: \sqrt{ } 4: \sqrt{ } 7: \sqrt{ } 9: \sqrt{ } 12: \sqrt{ } 13$. At $90{ }^{\circ} \mathrm{C}$, a clear region of $L$ is evident by the reflections positioned at integral $\mathrm{q} / \mathrm{q}^{*}$ ratios of $1: 2: 3$. Cooling from $90^{\circ} \mathrm{C}$, or heating from 60 ${ }^{\circ} \mathrm{C}$, produces scattering patterns similar to that shown for $80^{\circ} \mathrm{C}$ in which both sets of reflections are observed. As shown more clearly in the inset in Figure 6, at 80 ${ }^{\circ} \mathrm{C}$ the principal peak is actually two closely spaced peaks, each corresponding to the $q^{*}$ of the respective $L$ or $\mathrm{C}_{2}$ phases. Extrapolation of the $\mathrm{T}$ dependence of $\mathrm{q}^{*}$ for each phase from the one-phase windows into the twophase window enabled the $q^{*}$ for each phase to be identified unambiguously within the two-phase window; the higher order reflections in the coexistence window could then be conclusively indexed. It is important to note that these transitions are reversible with temperature and stable with prolonged annealing $(>500 \mathrm{~h})$. Therefore, we conclude that the observed behavior reflects equilibrium structures and transition temperatures.

Second, the $\mathrm{S}_{2}{ }^{\mathrm{bcc}}$ window that occurs in DBP has been replaced by a region of inverted fcc-packed spheres $\left(\mathrm{S}_{2} \mathrm{fcc}^{\mathrm{cc}}\right.$. An example of the SAXS pattern produced for $\mathrm{S}_{2}{ }^{\mathrm{fcC}}$ is shown for a $\phi=0.26$ solution in DEP at $10{ }^{\circ} \mathrm{C}$ in Figure 7. The pattern shows excellent agreement between the observed peaks and the allowed reflections at $\sqrt{ } 3: \sqrt{ } 4: \sqrt{ } 8: \sqrt{ } 11: \sqrt{ } 12: \sqrt{ } 16: \sqrt{ } 19: \sqrt{ } 20: \sqrt{ } 24: \sqrt{ } 27$. Superimposed onto the scattering arising from the lattice are three (spherical) form factor oscillations with minima 


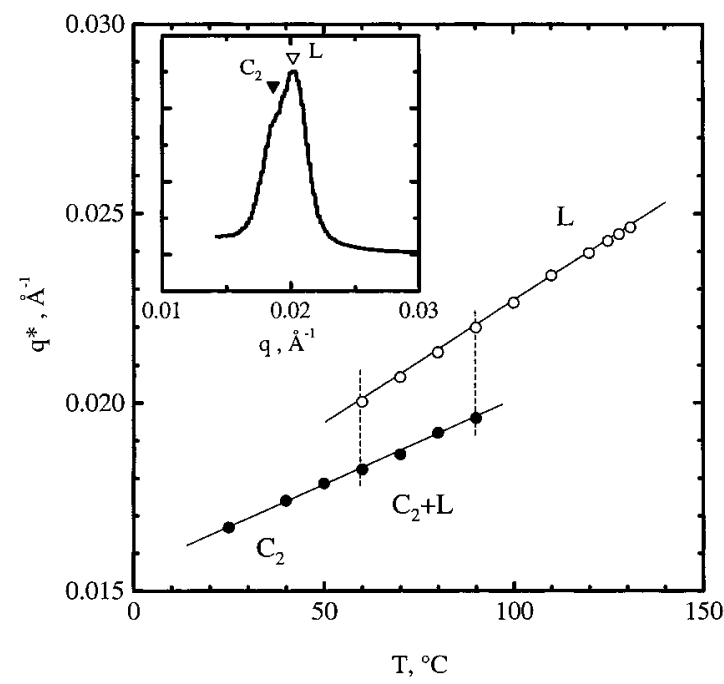

F igure 6. Temperature dependence of $\mathrm{q}^{*}$ for $\mathrm{C}_{2}$ (filled circles) and $L$ (open circles) for a $\phi=0.50 \mathrm{DEP}$ solution. The solid lines are a guide to the eye. The dashed lines indicate the phase boundaries. The inset shows a SAXS profile at $80{ }^{\circ} \mathrm{C}$ for the solution denoting the positions of the two closely spaced principal reflections of the $\mathrm{C}_{2}$ and $\mathrm{L}$ phases.

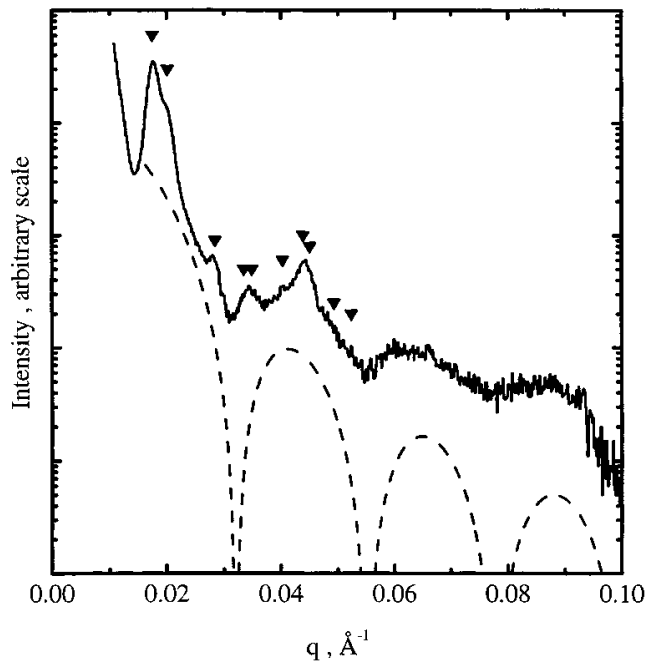

Figure 7. Representative SAXS profiles of a $\phi=0.26$ DEP solution at $20^{\circ} \mathrm{C}$. The filled triangles correspond to possible fcc reflections at $q / q^{*}$ spacings of $\sqrt{ } 3: \sqrt{ } 4: \sqrt{ } 8: \sqrt{ } 11: \sqrt{ } 12: \sqrt{ } 16$ : $\sqrt{ } 19: \sqrt{ } 20: \sqrt{ } 24$. The dashed line corresponds to a spherical form factor for $R=140 \AA: P(q, R)=\left[3(\sin (q R)-q R \cos (q R)) /(q R)^{3}\right]^{2}$.

near $0.03,0.055$, and $0.075 \AA^{-1}$. This corresponds to a hard-sphere radius-approximately equal to the micelle core radius since the coronal blocks significantly overlapof ca. $140 \AA$ as shown by the agreement with the calculated form factor for this radius (dashed line in Figure 7). Assuming that the micellar structure is approximately independent of concentration, the micelle therefore has a core radius of 140 and a $50 \AA$ coronal layer (190 ^̊ hydrodynamic radius). Interestingly, solutions near the $\mathrm{C}_{2} / \mathrm{S}_{2}$ fcc boundary display thermotropic OOTs between these two structures. For example, Figure 8 displays the SAXS patterns for the $\phi=0.26$ solution in DEP at several temperatures. At or below $90{ }^{\circ} \mathrm{C}, \mathrm{I}$ (q) displays the characteristic fcc pattern, but upon heating to $100^{\circ} \mathrm{C}$, the two cl osely spaced peaks at $\sqrt{3}: \sqrt{ } 4 \mathrm{q} / \mathrm{q}^{*}$ transform into a single peak, with clear higher order reflections devel oping at $\sqrt{3}$ and $\sqrt{ } 7 \mathrm{q} / \mathrm{q}^{*}$, indicative of $\mathrm{C}_{2}$. At $120^{\circ} \mathrm{C}$ the higher order reflections

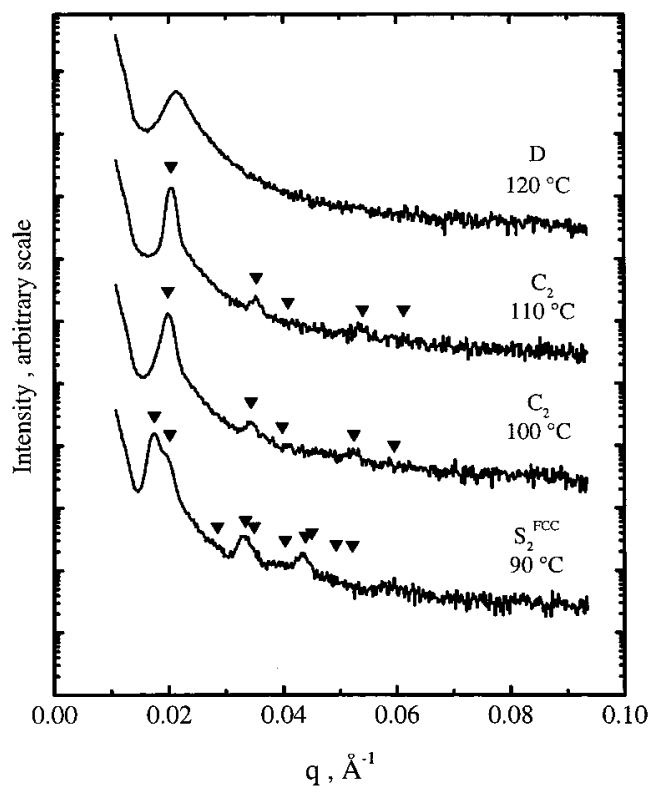

Figure 8. Representative SAXS profiles as a function of temperature for a $\phi=0.26 \mathrm{DEP}$ solution. The filled triangles correspond to possible reflections at $q / q^{*}$ spacings of $1: \sqrt{ } 3: \sqrt{ } 4$ : $\sqrt{ } 7: \sqrt{ } 9$ for $C_{2}$ and $\sqrt{ } 3: \sqrt{ } 4: \sqrt{ } 8: \sqrt{ } 11: \sqrt{ } 12: \sqrt{ } 16: \sqrt{ } 19: \sqrt{ } 20: \sqrt{ } 24: \sqrt{ } 27$ for $\mathrm{S}_{2}{ }^{\text {fcc }}$.

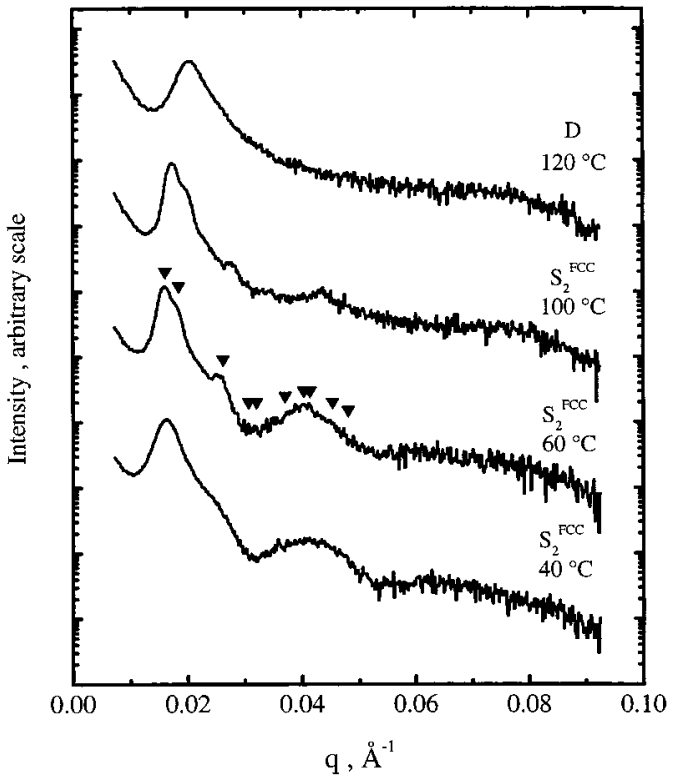

Figure 9. Representative SAXS profiles as a function of temperature for a $\phi=0.20 \mathrm{DEP}$ solution. The filled triangles correspond to possible reflections at $q / q^{*}$ spacings of $\sqrt{ } 3: \sqrt{ } 4$ : $\sqrt{ } 8: \sqrt{ } 11: \sqrt{ } 12: \sqrt{ } 16: \sqrt{ } 19: \sqrt{ } 20: \sqrt{ } 24: \sqrt{ } 27$ for $\mathrm{S}_{2}^{\text {fcc. }}$.

disappear, and the q* peak broadens considerably upon traversing the ODT.

Third, at low polymer concentrations, a "reentrant" thermotropic ODT is observed for a $\phi=0.20$ solution near the lyotropic $\mathrm{S}_{2}{ }^{\mathrm{fcc}} \rightarrow$ micelle transition. The SAXS pattern at $40{ }^{\circ} \mathrm{C}$ is dominated by the micellar form factor, which displays several broad oscillations (Figure 9). The absence of higher order structure factor peaks indicates that the solution is a disordered suspension of interacting micelles. U pon heating to $60^{\circ} \mathrm{C}$, the twodimensional scattering image (not shown) develops a "spotty", anisotropic pattern often indicative of the development of a large grain cubic morphology. The radial-averaged one-dimensional pattern in Figure 9 

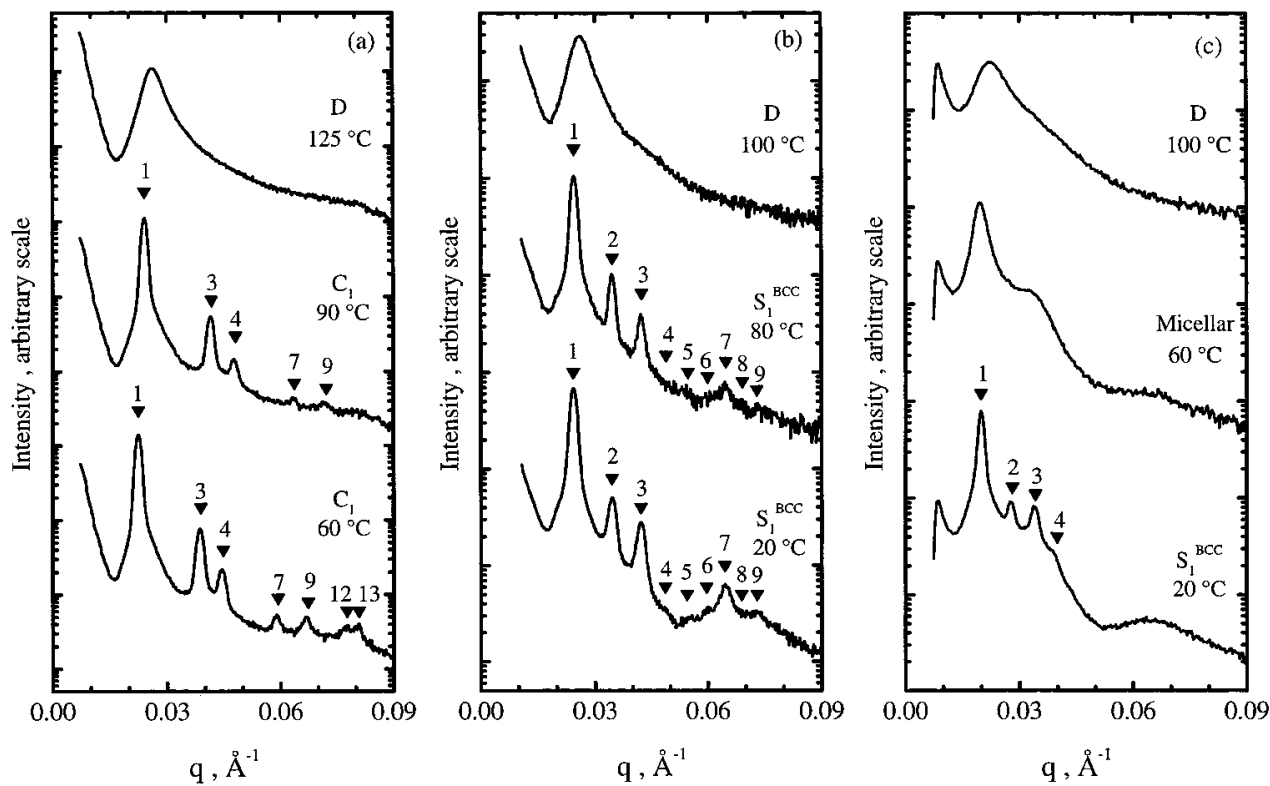

Figure 10. Representative SAXS profiles as a function of temperature for C14 solutions: (a) $\phi=0.48$; (b) $\phi=0.35$; (c) $\phi=0.17$. The filled triangles correspond to possible reflections at $q / q^{*}$ spacings of the indicated squared ratios.

shows that an fcc structure has developed. Further heating disrupts the $\mathrm{S}_{2}^{\text {fcc }}$ phase at $120{ }^{\circ} \mathrm{C}$ and the solution disorders. Thus, the phase sequence upon heating is micellar $\rightarrow \mathrm{S}_{2}{ }^{f c c} \rightarrow$ disordered. It should be noted that both ODTs are thermoreversible and that the micellar solution is classified as a disordered phase.

Tetradecane Phase Diagram. In contrast to DBP and DEP, n-tetradecane (C14) is an isoprene-selective solvent. Accordingly, the phase diagram of $\mathrm{SI}(11-21)$ in C14, shown in Figure 1, is markedly different. Compared to the six normal and inverted ordered equilibrium structures observed in DBP and DEP, only three normal structures occur in C14. In the most concentrated solutions, a small $\mathrm{G}_{1}$ region is presumed to occur due to the presence of this phase at low temperatures in the neat block copolymer. SAXS and static birefringence indicate that this window must be restricted to $\phi$ $>0.98$. A relatively large window of $\mathrm{C}_{1} \rightarrow \mathrm{D}$ transitions occurs for $0.48 \leq \phi \leq 0.98$ at progressively lower temperatures. Representative $\mathrm{C}_{1}$ SAXS patterns are shown for $\phi=0.48$ in Figure 10a. Well-ordered hexagonal-packed cylinders are easily identified at $60^{\circ} \mathrm{C}$ due to the presence of seven reflections located at $\mathrm{q} / \mathrm{q}^{*}$ ratios of $1: \sqrt{ } 3: \sqrt{ } 4: \sqrt{ } 7: \sqrt{ } 9: \sqrt{ } 12: \sqrt{ } 13$. Heating the sample produces a gradual decrease in the higher order peak intensities associated with a loss in long-range order. Ultimately, at $125{ }^{\circ} \mathrm{C}$ the sample disorders; as the higher order peaks disappear, the principal peak abruptly decreases in intensity and the fwhm triples.

F or $\phi<0.48$ the solutions are not birefringent, which suggests the formation of a cubic phase. SAXS experiments indicate that bcc spheres exist between $\phi=0.48$ and $\phi=0.15$. Examples are shown for $\phi=0.35$ and $\phi=$ 0.17 solutions in parts $b$ and $c$ of $F$ igure 10 , respectively. For $\phi=0.35$ at $20^{\circ} \mathrm{C}$, scattering arises from both the structure factor and the form factor as indicated by the sharp Bragg reflections at low q superimposed onto the broad oscillation evident at higher $\mathrm{q}$. The reflections positioned at $1: \sqrt{ } 2: \sqrt{ } 3: \sqrt{ } 7: \sqrt{ } 9 q^{*}$ are attributed to a bcc lattice; the expected peaks at $\sqrt{ } 4: \sqrt{ } 5: \sqrt{ } 6 q^{*}$ are diminished presumably due to the form factor minimum. In this case, the presence of the $\sqrt{ } 7 q^{*}$ reflection allows the possibility of a simple cubic (sc) lattice to be discounted. The bcc lattice remains upon heating until between 80 and $100{ }^{\circ} \mathrm{C}$ where the structure factor scattering abruptly disappears due to the ODT. The $\phi$ $=0.17$ solution at $20{ }^{\circ} \mathrm{C}$ (Figure 10C) also displays scattering from the structure factor and form factor. The scattering peaks at $1: \sqrt{ } 2: \sqrt{ } 3 q^{*}$ also indicate a cubic lattice which is presumably bcc. The higher order peaks disappear upon heating this solution, leaving scattering from a weak liquidlike structure factor peak and the form factor. This is attributed to an ODT at $55^{\circ} \mathrm{C}$ from the $\mathrm{S}_{1}$ bcc phase to a liquidlike phase of spherical micelles. The structure and form factor scattering gradually decrease in intensity with increasing temperature, until above $100{ }^{\circ} \mathrm{C}$ only a broad structure factor peak re mains.

Dilute solution measurements of SI(11-21) in C14 confirm the presence of micellar-like aggregates at low temperature that melt into single chains near $100{ }^{\circ} \mathrm{C}$. In Figure $4 \mathrm{~b}$, the temperature dependence of the scattered light intensity for a $\phi=0.015$ solution shows significant scattering bel ow $70^{\circ} \mathrm{C}$, followed by an abrupt increase to a maximum intensity at $80^{\circ} \mathrm{C}$ and then a rapid decrease to near zero by $100^{\circ} \mathrm{C}$. The temperature dependence of the hydrodynamic radius, shown in Figure $4 a$, foll ows a similar trend. At $40^{\circ} \mathrm{C}$, the radius in C14 is only slightly larger than the micelle formed in DBP and DEP. This may be attributed to the difference in micellar structure between the solvents. In DBP and DEP, "crew-cut" micelles are formed with the longer PI blocks forming the core and the shorter PS blocks the corona. In C14, the isoprene selectivity dictates a "hairy" micelle in which the PI forms the corona and the core is PS. I ncreasing the temperature produces a near 10-fold increase in $\mathrm{R}_{\mathrm{h}}$ before abruptly decreasing to ca. $60 \AA$ by $100{ }^{\circ} \mathrm{C}$. The decrease in $R_{h}$ and scattered intensity near $100{ }^{\circ} \mathrm{C}$ mark the $\mathrm{cmt}$ for this system. The anomal ous increasing intensity and $R_{h}$ near the cmt is not fully understood, but similar behavior has been observed in other diblock copolymer micellar solutions. ${ }^{21,42-44}$ It probably reflects the incipient phase separation of trace amounts of homopolymer. Finally, we draw attention to the observation that the cmt for $\mathrm{SI}(11-21)$ in $\mathrm{C} 14$ is again in excellent agreement 


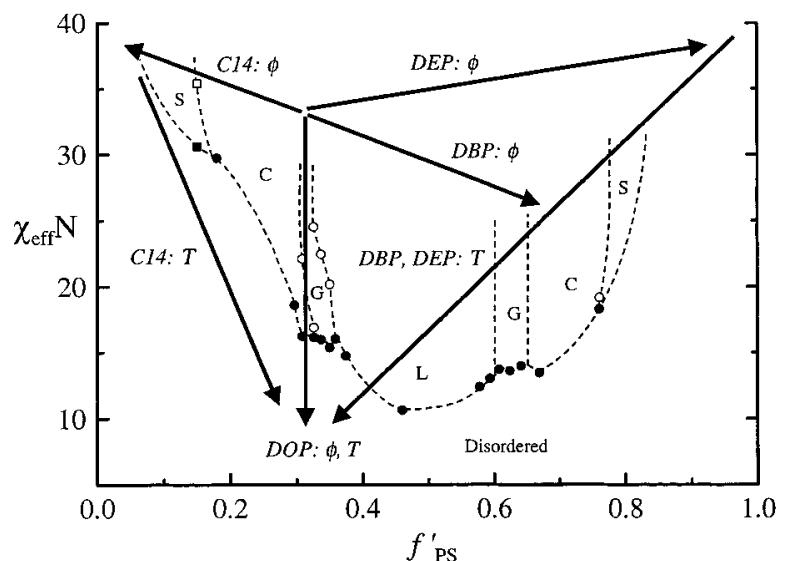

Figure 11. Phase trajectories of SI (11-21) in DOP, DBP, DEP, and C14. The open and closed symbols correspond to OOTs and ODTs, respectively, determined by Khandpur et al. ${ }^{45}$ (circles) and Ryu et al. ${ }^{46}$ (squares) for SI copolymers, with the dashed lines marking the estimated phase boundaries. The trajectories start at the estimated segregation of neat SI(1121) at $0{ }^{\circ} \mathrm{C}$.

with the extrapolated TODT for concentrations between $\phi=0.3$ and 0.4 (Figure 1).

\section{Discussion}

The discussion is divided into three parts. First, we demonstrate how many of the features of the phase diagrams in Figure 1 can be understood via the concept of trajectories across the melt phase map. Second, we examine those features that are not easily explained in this way. Finally, we consider the dependence of the principal domain spacing, $\mathrm{d}^{*}$, on $\phi, \mathrm{T}$, and structure.

Trajectory Approach. Many aspects of the phase behavior of SI (11-21) in the four solvents are reminiscent of neat block copolymers, particularly in terms of the structures that are observed and the "topology" of the diagram (i.e., the sequence of phases upon changing $\phi$ or T). This suggests using the well-established principles that govern the melt phase behavior of diblock copolymers as a basis for understanding their solutions. Previously, we invoked the qualitative notion of trajectories across the SI melt phase map upon varying $\phi$ or $T$ to describe the phase behavior in concentrated solutions of SI(11-21) in DOP and DBP. ${ }^{9}$ Here, we extend this concept to examine more closely the effect of varying the solvent selectivity. This is illustrated in Figure 11, where six trajectories associated with the expected phase behavior in each of the investigated solvents are superimposed onto the melt phase map, presented in terms of an effective PS volume fraction ( $f^{\prime} p s$ ) and degree of segregation $\chi_{\text {eff }} \mathrm{N}$. The melt phase map is drawn based on extensive results for SI melts, 45,46 with the polystyrene composition as the independent variable, a PS reference volume for $\mathrm{N}$, and $\chi_{\text {eff }}$ from eq 2 . As a common reference, the trajectories originate at the estimated segregation of neat SI (11-21) at $0{ }^{\circ} \mathrm{C}$.

Of central importance is the effect of the diluent on the effective segregation between the two blocks. If the solvent is perfectly neutral $\left(\chi_{\mathrm{AS}}=\chi_{\mathrm{BS}}\right)$, then the solvent is distributed equally between the microdomains. The enthalpic contribution to the segregation can then be expressed as a single effective interaction parameter of the form

$$
\chi_{\mathrm{eff}} \sim \phi^{\beta} \chi
$$

where $\chi$ is the monomer-monomer interaction parameter. When $\beta=1$, eq 3 is the well-known "dilution approximation" that SCMF theory anticipates will describe the solution phase behavior (i.e., by replacing the melt degree of segregation $\chi \mathrm{N}$ by $\phi \chi \mathrm{N}) .{ }^{14-18}$ The effect of added neutral solvent is to simply "dilute" the monomer-monomer contacts, thereby reducing the effective segregation; this is analogous to increasing the temperature in the melt and consequently corresponds to a vertical trajectory across the phase map (i.e., decreasing $\chi_{\text {eff }} \mathrm{N}$ at constant $\left.\mathrm{f}^{\prime} \mathrm{PS}\right)$. However, this is not exactly the case with $\mathrm{SI}(11-21)$ in DOP, as the $C_{1}$ phase is diminished upon dilution due to competing concentration scalings of $\chi_{\text {eff }}$ at the OOT $\left(\chi_{\text {OOT }} \sim \phi^{-1}\right)$ and ODT ( ODT $_{\text {O }} \sim \phi^{-1.4}$ ).

In the other limit, a strongly selective solvent $\left(\chi_{\mathrm{AS}} \ll\right.$ $\chi_{\mathrm{BS}}$ ) will preferentially segregate into the A domains. In this case, the effective segregation cannot be readily reduced to a simple function of the three $\chi$ parameters (in the spirit of eq 3 ) and may depend separately on factors such as $\phi, f$, and $N$. H owever, if $\chi_{B S}$ is sufficiently large, the solvent will be repelled from the $A-B$ interface. To minimize the $B-S$ contacts, the interfacial area per chain is decreased, and thus the degree of segregation is increased relative to the melt state. This is analogous to the mechanism presented by Matsen to describe the increase in degree of segregation of an $A-B$ diblock upon addition of a high molecular weight $A$ homopolymer; in this case the small entropy of mixing leads to the repulsion of the homopolymer from the interface. ${ }^{47}$ Based on the two limits of a strongly selective and neutral solvent, differing degrees of selectivity will then move the system toward lesser or greater degrees of segregation at a given concentration. Ultimately, it is the degree of segregation that determines the ordered state stability, and thus varying the solvent selectivity will either increase or decrease the region of stable ordered structures. ${ }^{16}$

For our systems we are able to directly determine the effect of increasing the solvent selectivity on the ordered state stability. This effect is shown in Figure 11 as the vertical component of the concentration-dependent trajectories for DBP, DEP, and C14. Based on previous work, DBP is slightly PS selective and hence decreases the effective segregation relative to the melt at $0{ }^{\circ} \mathrm{C}$; the vertical component of the trajectory is thus downward, toward weaker segregation. ${ }^{22,23}$ Note that although the segregation relative to the melt at $0{ }^{\circ} \mathrm{C}$ is weakened, it is increased relative to the neutral solvent DOP at the same $\phi$. Therefore, for $\phi<0.6$ the addition of DBP stabilizes ordered structures which are disordered in DOP. As DEP is more PS selective, its trajectory is to larger $\chi_{\text {eff }} \mathrm{N}$. Consequently, an increased window of ordered structures is observed, as indicated by the increase in TODT for DEP relative to DBP. F urthermore, both DEP and $\mathrm{C} 14$ are expected to increase the segregation relative to the melt state at 0 ${ }^{\circ} \mathrm{C}$. This will be shown subsequently to have a significant effect on the $\phi$ dependence of the domain spacing and the exact phase sequence upon dilution.

The effect of preferential partitioning can be approximately accounted for via a renormalized block volume fraction, $f^{\prime}$, based on the relative microdomain volumes. For neutral solvents, $f^{\prime}=f$, but for a completely partitioned solvent, $\mathrm{f}^{\prime}=\mathrm{f} \phi+(1-\phi)$. Accordingly, addition of a selective solvent corresponds to a trajectory with a substantial horizontal component to 
higher block volume fractions, analogous to increasing the relative block length. The trajectory reflects the change in the spontaneous curvature of the interface due to preferential swelling of the A block, resulting in a series of Iyotropic OOTs. Note, however, that the driving force for OOTs as a result of changes in $\mathrm{f}$ or $\phi$ are quite different. The former reflects only the minimization of chain stretching, whereas the latter depends strongly on the degree of partitioning and hence the selectivity of the solvent. Ultimately with sufficient solvent the swelling disperses the ordered structures into noninteracting micelles. Although the mechanisms are different, the qualitative correlation between these parameters when the solvent is selective is clear.

This concept is well illustrated by the correspondence between the phase sequence in DBP and DEP at low temperature upon decreasing $\phi$ from the melt state and the phase transitions that are expected upon increasing the PS block length starting at the composition of SI$(11-21)\left(f_{P S}=0.31\right)$ in the melt phase map. In DBP the observed phase sequence is $\mathrm{G}_{1} \rightarrow \mathrm{L} \rightarrow \mathrm{G}_{2} \rightarrow \mathrm{C}_{2} \rightarrow \mathrm{S}_{2}$ bcc $\rightarrow \mathrm{D}$, and in DEP it is $\mathrm{G}_{1} \rightarrow \mathrm{L} \rightarrow \mathrm{L}+\mathrm{C}_{2} \rightarrow \mathrm{C}_{2} \rightarrow \mathrm{S}_{2} \mathrm{fcc} \rightarrow$ $D$ ( $D$ includes the micellar phase). The replacement of $\mathrm{G}_{2}$ by $\mathrm{L}+\mathrm{C}_{2}$ coexistence in DEP and the exact cubic packing (bcc vs fcc) are not captured in the trajectory approach. In C14, the horizontal trajectory toward smaller PS block lengths is consistent with the phase behavior observed in this isoprene-selective solvent: the sequence $\mathrm{G}_{1} \rightarrow \mathrm{C}_{1} \rightarrow \mathrm{S}_{2}$ bcc $\rightarrow \mathrm{D}$ is expected and observed. The greater selectivity of DEP relative to that of DBP is demonstrated by the decrease in $\phi_{\text {Оот }}$ at low temperature needed to produce the equival ent morphological transition. This shift is primarily due to increased partitioning of solvent to the PS domains, resulting in larger changes in $f^{\prime}$ for a given $\phi$. DBP is only slightly selective at low temperature and does not partition completely into the PS domains.

The situation upon heating is more complicated. The solvent will generally become less selective and therefore redistribute more uniformly between microdomains. Consequently, the phase behavior follows a diagonal trajectory back across the phase map toward structures dictated by the block copolymer composition (i.e., $f^{\prime} \rightarrow$ $f$ upon heating). As a result, a rich variety of thermotropic OOT and ODT transitions are accessible. For example, the phase sequence upon heating sol utions at high $\phi$ illustrates the crossover from morphologies with interfacial curvature determined by both the partitioned solvent and $f$ at low temperature (e.g., $L$ ), to that controlled by only $f$ at high temperature where the phase sequence mi mics the melt copolymer $\left(\mathrm{G}_{1} \rightarrow \mathrm{C}_{1} \rightarrow\right.$ D). In DBP, the decrease of Tоот with decreasing $\phi$ for the $C_{1} \rightarrow G_{1}$ transition is similar to the effect of dilution with DOP, but at lower temperature the $\mathrm{L} \rightarrow \mathrm{PL} / \mathrm{G}_{1}$ $T_{\text {оот }}$ is al most concentration independent. This can be compared to the same region in the DEP phase diagram in which both $\mathrm{T}_{\text {OOT'S }}$ increase with decreasing $\phi$, reflecting the greater contribution of partitioned solvent due to an increased solvent selectivity. The similarity of TODT for solutions with $\phi>0.8$ for both the neutral solvent DOP and the increasingly PS-selective solvents DBP and DEP confirms the dominant contribution of (diluted) block interactions. Decreasing $\phi$ corresponds to low-temperature "starting points" at larger values of $f^{\prime}$, such that upon heating the trajectory crosses the ODT directly from morphologies not found in the melt state, e.g., $\mathrm{L} \rightarrow \mathrm{D}$ transitions.
The qualitative success of the trajectory approach in describing the role of a temperature-dependent solvent selectivity suggests that it may offer a promising route to anticipating the phase behavior of any block copolymer/ solvent system based on the neutral and selective trajectories. In particular, TODT can be semiquantitatively predicted over the entire concentration range based on knowledge of only the cmt and melt T ODT. As shown in the selective solvent phase diagrams (Figure $1)$, the concentration dependence of TODT reflects the superposition of a concentration-independent value equal to the cmt for semidilute concentrations and the dependence found for neutral solvents in more concentrated solutions. It is not surprising that the $\mathrm{cmt}$ in dilute solution matches the ODT in moderately concentrated solutions because they both represent the point at which the block-solvent interactions have sufficiently weakened such that the chains can disperse. Furthermore, these results demonstrate the utility of the $\mathrm{cmt}$ as a simple measure of the selectivity of a given solvent.

Novel Features. As noted above, several novel features emerge ( $L+C_{2}$ coexistence, bcc vs fcc micellar packing, "reentrant" thermotropic ODT) that are not captured in the trajectory approach. These features are correlated with increasing solvent selectivity as they only become apparent in the more sel ective solvent DEP.

The occurrence of coexisting phases is quite common in aqueous surfactant systems and in some block copolymers, ${ }^{28}$ particularly the poly(ethylene oxide)poly(butylene oxide) block copolymers in water. ${ }^{30-33}$ The observation of coexistence is not surprising in itself, given that for a two-component system one expects coexistence regions along each phase boundary. However, for almost all the transitions examined here the coexistence window is very small (i.e., $\leq 1^{\circ} \mathrm{C}$ ), whereas near the $L / C_{2}$ window in DEP the interval is as much as $40{ }^{\circ} \mathrm{C}$ and $\Delta \phi \approx 0.1$ wide. This is presumably attributable to the subtle balance of chain stretching, packing frustration, and conformational asymmetry that ultimately determines the precise location of the L/G/C phase boundaries. ${ }^{48-51}$ Experiment ${ }^{39,40}$ and theory ${ }^{48-50}$ show that the $G$ phase is only stable in weakly segregated block copolymers; it is destabilized at stronger segregations due to a heightened free energy penalty of packing frustration in both the minor and major domains. Hajduk et al. attributed the near absence of $\mathrm{G}$ in aqueous solutions of a PEO-poly(ethylethylene) diblock to the increase in segregation imparted by the strong selectivity of the water for PEO. ${ }^{28}$ Similar arguments have been reported for amphiphilic suspensions. ${ }^{52,53}$ We therefore hypothesize that in the case of SI (11-21) the $L+C_{2}$ coexistence window occurs due to the instability of $G_{2}$ at stronger segregations induced by the selectivity of DEP at low temperature. As the segregation decreases with increasing temperature, $\mathrm{G}_{2}$ becomes stable as the packing frustration is relieved, and the thermodynamic expense weakens. In DBP, the coexistence window is absent, but we postulate its existence at temperatures below $0{ }^{\circ} \mathrm{C}$ (i.e., at stronger segregations).

The inverted cubic phase adopts a bcc packing in DBP and $\mathrm{C} 14$ but an fcc packing in DEP. This interesting observation is broadly consistent with some previous reports, $26,27,54,55$ where this behavior is attributed to the range of the intermicellar interaction potential. McConnell and co-workers empl oyed SI diblock copolymers 
of various $f$ and $\mathrm{N}$ in decane. ${ }^{26,27}$ The key variable was the ratio of the $\mathrm{PI}+$ solvent coronal layer thickness $\mathrm{L}$ to the PS core radius $R_{c}$. For relatively thin coronal layers $\left(L / R_{c}<1.5\right)$, the repulsive interactions are shortranged, and thus like hard spheres the micelles select the close-packed fcc lattice. Conversely, micelles with thicker coronal layers $\left(L / R_{c}>1.5\right)$ have longer-ranged, "softer" interactions which favor the bcc arrangement. This argument is consistent with our observation of the bcc phase in the isoprene-selective solvent C14, since here we estimate $L / R_{c}>2$, based on $R_{h} \approx L+R_{c} \approx 280$ $\AA$ and $R_{c} \approx 90 \AA$. Similarly, in DEP we estimate $L / R_{c} \approx$ 0.4 from $R_{h} \approx L+R_{c} \approx 190 \AA$ and $R_{c} \approx 140 \AA$, and thus fcc is expected. However, this argument is not sufficient to explain the difference in cubic packing between DBP and DEP because whereas the transitions in the work by McConnell were produced by varying the relative block lengths; here only the degree of styrene selectivity has changed. The $R_{h}$ for micelles in DEP and DBP is the same, and it is hard to imagine that $L / R_{c}$ could differ by a factor of 4 from DEP. Therefore, it appears that the reason for the $\mathrm{S}_{2}$ bcc phase in DBP is not captured by the McConnell and Gast picture. In a related study, Hamley and co-workers investigated a thermotropic transition from a bcc to an fcc cubic lattice in an aqueous solution of a PEO-PBO diblock copolymer. ${ }^{54,55}$ At low temperature PEO is well solvated by water, and consequently the micelles adopt a bcc lattice due to the larger coronal layer of the hydrophilic PEO block relative to the PBO core. U pon increasing the temperature, the water becomes a poorer solvent for the PEO. Detailed static and dynamic light scattering experiments reveal that this results in a contraction of the coronal layer accompanied by an increase in the core radius due to an increased aggregation number. ${ }^{56}$ Consequently, the intermicellar interactions become more short-ranged, and the structure adopts a fcc lattice. Although this illustrates the effect of a changing solvent quality on the micellar structure, our system differs in the sense that the PS coronal chains are wellsolvated over the entire temperature range, and it is mainly the degree of selectivity toward core PI chains that influences the micellar packing.

The last issue is the dependence of the structural length scale, $d^{*}$, upon concentration and temperature. It was previously reported for SI(11-21) in the neutral solvent DOP that the gyroid domain spacing $\left(d_{211}\right)$ was found to decrease with added solvent as $\mathrm{d}_{211} \sim \phi^{1 / 3}$. ${ }^{2}$ This agrees with the more general results anticipated by SCMF theory ${ }^{14,15}$ and those found in earlier experimental studies ${ }^{4}$ of block copolymers in neutral solvents. This result is a consequence of the decreased segregation which enables the chains to increase their lateral spacing and thus to decrease their stretching normal to the interface. When the solvent is selective, the balance of interfacial area and chain stretching is more complicated. For example, $d^{*}\left(d_{100}\right)$ in the $L$ phase for $\mathrm{SI}(11-21)$ in DBP and DEP at several concentrations and temperatures is shown in a double-logarithmic format in Figure 12 along with the determined powerlaw scalings of $\mathrm{d}_{100} \sim \phi^{\alpha}$. In DBP (Figure 12a), $\mathrm{d}_{100}$ decreases with increasing $T$ for all concentrations, as expected; the entropic penalty for chain stretching increases with $\mathrm{T}$. At a given temperature, $\mathrm{d}_{100}$ decreases with added solvent. At $20{ }^{\circ} \mathrm{C} \mathrm{d}_{100} \sim \phi^{0.18}$, a slightly weaker dependence than $\phi^{1 / 3}$, but the exponent increases with temperature until at $80{ }^{\circ} \mathrm{C}$ the results correspond
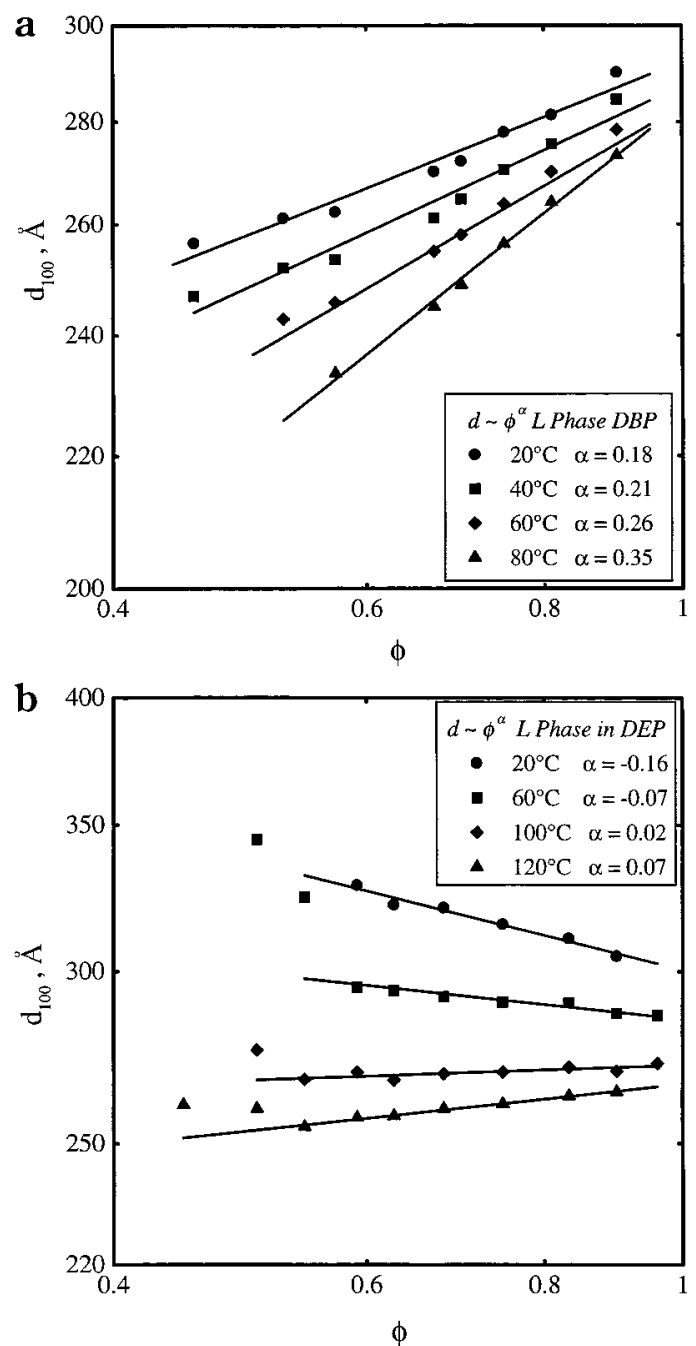

Figure 12. Double-logarithmic plot of the concentration dependence of the $\left(\mathrm{d}_{100}\right)$ domain spacing of the lamellar phase for various temperatures: (a) DBP solutions; (b) DEP solutions. The solid lines correspond to the estimated power-law dependence $\left(\mathrm{d} \sim \phi^{\alpha}\right)$. Note that the two lowest concentrations at 60 and $120{ }^{\circ} \mathrm{C}$ and the lowest concentration at $100{ }^{\circ} \mathrm{C}$ of the DEP solutions were not included in the regression. The uncertainty in $d_{100}$ is less than $\pm 5 \AA$.

to the addition of neutral solvent. In DEP, however, the domain spacing exhibits a crossover from increasing with added solvent at $20^{\circ} \mathrm{C}\left(\mathrm{d}^{*} \sim \phi^{-0.16}\right)$ to decreasing at $120^{\circ} \mathrm{C}$ as $\mathrm{d}^{*} \sim \phi^{0.07}$ (Figure $12 \mathrm{~b}$ ). This phenomenon is clearly attributed to the temperature-dependent solvent selectivity.

Banaszak and Whitmore have examined the scaling of $d^{*}$ for the $L$ phase in a selective solvent and find that the domain spacing decreases as $d^{*} \sim \phi^{0.2}$ in the weak segregation regime, but with a stronger dependence of $\mathrm{d}^{*} \sim \phi^{0.5}$ at strong segregation. ${ }^{13}$ However, they utilized a solvent that is good for both blocks $\left(\chi_{\mathrm{AS}}, \chi_{\mathrm{BS}}<0.5\right)$, and thus the results may not reflect the effect of a selective solvent in which one block is insoluble. Therefore, we have performed an SCMF analysis of $d^{*}$ in the $\mathrm{L}$ phase for an A-B diblock copolymer in a solvent (S) of varying selectivity using a previously established formalism. ${ }^{16}$ The model system is characterized by a block volume fraction $\mathrm{f}_{\mathrm{A}}=0.43$, a degree of polymerization $\mathrm{N}=400$, and interaction parameters $\chi_{\mathrm{AB}}=0.08$ and $\chi_{A S}=0.4$. These parameters reflect a lamellar forming block copolymer with $\mathrm{N}$ comparable to SI (11$21), \chi_{\mathrm{AB}} \approx \chi_{\mathrm{S}}$ at $25^{\circ} \mathrm{C}$, and $\chi_{\mathrm{AS}}$ of a typical good solvent. 


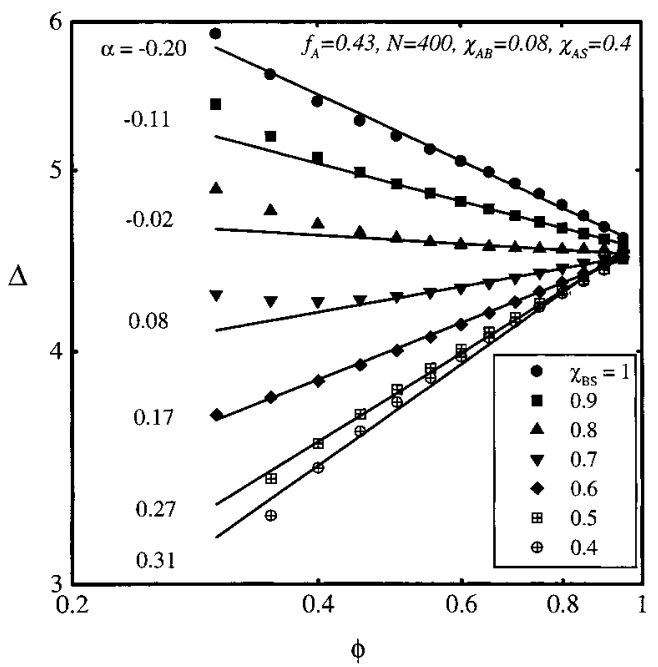

Figure 13. Doublelogarithmic plot of the concentration dependence of the dimensionless lamellar domain spacing ( $\Delta$ $\left.\equiv \mathrm{d}^{*} /\left(\mathrm{b}^{2} \mathrm{~N} / 6\right)^{1 / 2}\right)$ as determined using SCMF for an $\mathrm{f}_{\mathrm{A}}=0.43$ A-B diblock with $\mathrm{N}=400, \chi_{\mathrm{AB}}=0.08$, and $\chi_{\mathrm{AS}}=0.4$ for various values of $\chi_{\text {BS. }}$. The listed values of $\alpha$ correspond to the estimated power-law dependence of $\Delta$. Note that the two lowest concentrations at $\chi_{\mathrm{BS}}=0.9,0.8$, and 0.7 were not included in the regression.

The selectivity of the solvent is progressively increased from neutral, with $\chi_{\mathrm{BS}}=0.4$, to strongly selective, with $\chi_{\mathrm{BS}}=1$. For each set of the parameters, we minimize the free energy with respect to the domain spacing, $d * 1$ $\left(\mathrm{Nb}^{2} / 6\right)^{1 / 2}$ (b is the monomer statistical segment length). The results are shown in double-logarithmic format in Figure 13, along with the determined power-law exponents. We note that the $L$ phase for a given set of parameters may not correspond to the absolute minimum free energy state, especially at low concentrations and strong selectivities, but we are only interested here in the change in domain spacing. The results indicate that the theory captures the crossover in concentration scaling upon varying $\chi_{\mathrm{BS}}$. When the solvent is neutral, the domain period contracts roughly as $\phi^{1 / 3}$, but when the solvent is made more selective, the power-law exponent increases, eventually changing sign, until ultimately $\mathrm{d}^{*} \sim \phi^{-0.2}$ at $\chi_{\mathrm{BS}}=1$.

The mechanism behind this phenomenon may be more easily understood by examining the distribution of solvent between the microdomains. Figure 14 displays the concentration profiles of the lamellar phase at $\phi=$ 0.6 for three values of $\chi_{\mathrm{BS}}$. As expected, when the solvent is neutral, it is equally distributed between the $A$ and $B$ domains with a slight accumulation at the $A-B$ interface. ${ }^{6,14,18}$ Upon increasing $\chi_{\mathrm{BS}}$, several notable changes occur. First, the solvent partitions preferentially to the A domain due to the more favorable enthalpy of mixing. Complete partitioning is prevented by the large entropic penalty of demixing the solvent from the $B$ domain; even at $\chi_{\mathrm{BS}}=1$, there is still ca. $20 \%$ solvent within the B domain. Second, the increase in segregation to minimize the unfavorable B-S interactions is illustrated by the decrease in interfacial width between domains and consequently the area per chain. This requires an increase in chain stretching normal to the interface, resulting in an increase in $d^{*}$. At the strongest segregation of $\chi_{\mathrm{BS}}=1, \phi_{\mathrm{A}}$ even shows a slight maximum near the interface. This interesting result occurs because the A monomers preferentially accumulate at the interface in order to screen the less favorable $\mathrm{B}-\mathrm{S}$ contacts.

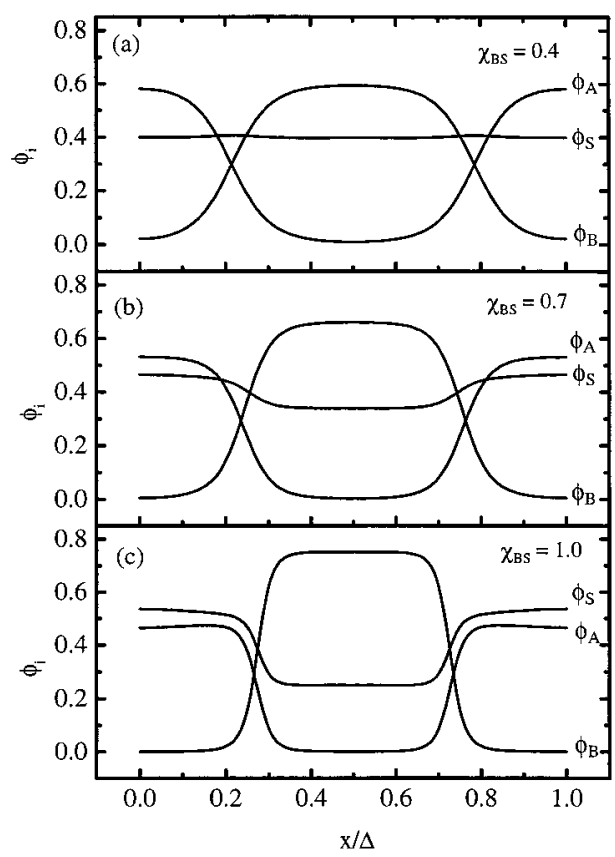

Figure 14. Predicted concentration profiles for the $\phi=0.6$ solutions in Figure 13 as a function of the dimensionless position $(x / \Delta)$ across a lamellar domain: (a) $\chi_{\mathrm{BS}}=0.4$; (b) $\chi_{\mathrm{BS}}$ $=0.7$; (c) $\chi_{\mathrm{BS}}=1$.

The effect of solvent selectivity on the concentration dependence of the structural length scale across the full concentration range is consistent with a crossover from behavior governed by block interactions at high concentration to solvent-polymer interactions at low concentration. Figure 15 shows $d^{*}$ over the entire concentration range of ordered structures at various temperatures for DBP (Figure 15a) and DEP (Figure 15b). In DBP, the lamellar period contracts with added solvent at 10 and $20^{\circ} \mathrm{C}$ as previously explained. However, once the structures invert, the addition of solvent produces a steady increase in $d^{*}$ at all temperatures. DEP solutions show an even stronger increase in $d^{*}$ upon formation of the inverted structures (note vertical scales of Figure 15b). This behavior reflects the ability of the inverted microstructure to increase the intermicelle distance by swelling the PS matrix without significantly altering the dimensions of the PI core. The swelling is evident by the $\mathrm{d}^{*} \sim \phi^{-1 / 3}$ concentration scaling for the $\mathrm{C}_{2}$ and $\mathrm{S}_{2}{ }^{\mathrm{fcc}}$ phases in DEP at 20 and $60^{\circ} \mathrm{C}$. However, this simple mechanism cannot capture the observed discontinuities in the concentration dependence of $d^{*}$ accompanying the lyotropic OOTs. In DBP, the $L \rightarrow G_{2}$ and $\mathrm{C}_{2} \rightarrow \mathrm{S}_{2}$ bcc transitions produce an abrupt $5 \%$ increase in $d^{*}$. F or DE $P, d^{*}$ is continuous across the $L$ $\rightarrow \mathrm{C}_{2}$ transition at 20 and $60{ }^{\circ} \mathrm{C}$ and the $\mathrm{L} \rightarrow \mathrm{G}_{2} \rightarrow \mathrm{C}_{2}$ transition at 100 and $120{ }^{\circ} \mathrm{C}$, but the $\mathrm{C}_{2} \rightarrow \mathrm{S}_{2}{ }^{\mathrm{fcc}}$ transition is discontinuous at all temperatures. Interestingly, this transition produces a $10 \%$ decrease in $d^{*}$ at $20^{\circ} \mathrm{C}$, but due to the different temperature dependences of $d^{*}$ for the $C_{2}$ and $S_{2}{ }^{f c c}$ phases, the decrease in d* diminishes with increasing temperature until at 100 ${ }^{\circ} \mathrm{C}$ a $5 \%$ increase is observed. The $\mathrm{C}_{1} \rightarrow \mathrm{S}_{1}$ bcc transition in C14 solutions produces a $10 \%$ decrease in $\mathrm{d}^{*}$ (not shown).

\section{Summary}

We have examined the phase behavior of a styreneisoprene (SI) diblock copolymer from the melt state to 

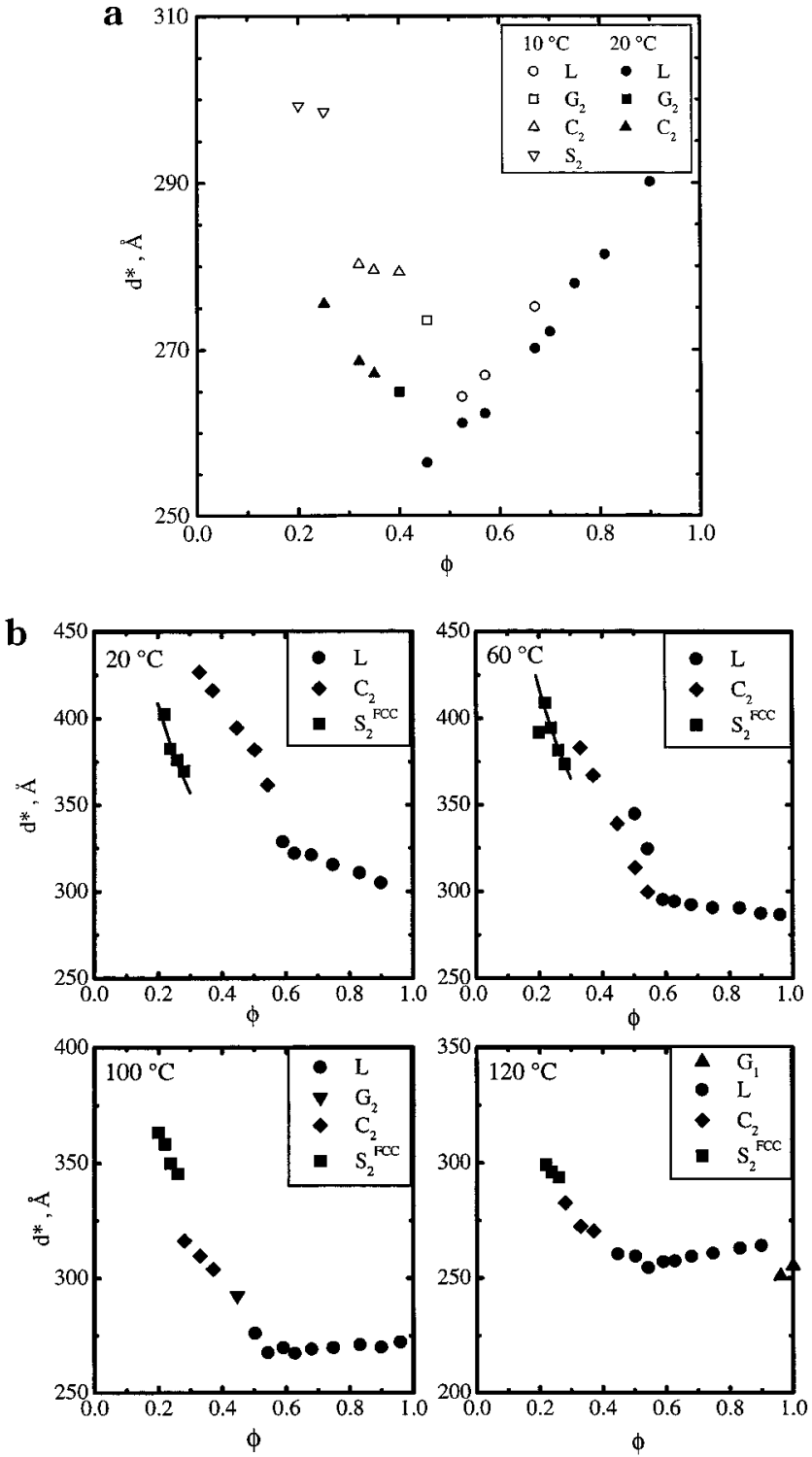

Figure 15. Concentration dependence of the domain spacing determined from the principal SAXS peak at various temperatures: (a) DBP solutions; (b) DEP solutions. The line extending through the values for $\mathrm{S}_{2}{ }^{\mathrm{fcc}}$ at 20 and $60{ }^{\circ} \mathrm{C}$ in DEP corresponds to $\mathrm{d}^{*} \sim \phi^{-1 / 3}$.

dilute solution in four solvents of varying thermodynamic selectivity for the two blocks; DOP is a neutral solvent, DBP and DEP are selective for styrene, and C14 is isoprene-selective. The phase behavior was examined using a combination of SAXS, static birefringence, and dynamic light scattering.

Upon heating, the undiluted block copolymer displays the phase sequence gyroid $\left(\mathrm{G}_{1}\right) \rightarrow$ hexagonal-packed cylinders $\left(C_{1}\right) \rightarrow$ disordered (D). Dilution with DOP decreases the order-order and order-disorder transition temperatures, and no new structures are observed. However, due to the failure of the dilution approximation for the ODT, the $\mathrm{C}_{1}$ window decreases with added solvent and ultimately disappears for polymer volume fractions $\phi<0.65$. Upon addition of DBP, a larger window of ordered states is revealed. Progressive lyotropic transitions to regions of lamellae ( $\mathrm{L}$ ), inverted gyroid $\left(\mathrm{G}_{2}\right)$, inverted hexagonal-packed cylinders $\left(\mathrm{C}_{2}\right)$, and inverted body-centered-cubic spheres $\left(\mathrm{S}_{2} \mathrm{bcc}\right)$ are found, as well as thermotropic transitions between them and to the disordered state. Addition of the increasingly selective solvent DEP produces similar transitions, although the ODTs occur at much higher temperatures. The C14 solutions display markedly different phase behavior-only $\mathrm{G}_{1}, \mathrm{C}_{1}$, and $\mathrm{S}_{1}$ bcc ordered phases are observed-due to the asymmetry in block copolymer composition. At dilute concentrations in all three selective solvents, the micelles disperse into polymer chains above the critical micelle temperature (cmt).

The sequence of phase transitions as a function of temperature (T) and concentration $(\phi)$ can be qualitatively anticipated using the concept of diagonal trajectories across the phase map ( $T$ vs composition f)) for neat block copolymers. The addition of a neutral solvent is analogous to increasing $T$ or a reduced effective interaction parameter $\left(\chi_{\text {eff }}\right)$, and thus a "vertical" trajectory, i.e., at constant $\mathrm{f}$. On the other hand, $\chi$ between a highly sel ective solvent and the unfavorable block may exceed that between the two monomers. This has the effect of increasing the segregation between microdomains and consequently the ordered state stability. Solvents with varying degrees of selectivity can therefore move the system to either lesser or greater degrees of segregation. Additionally, the unequal partitioning of a selective solvent between the block domains corresponds to a "horizontal" trajectory because the resulting lyotropic phase transitions can be largely accounted for using a renormalized block volume fraction ( $\left.f^{\prime}\right)$ based on the swollen block volume. However, upon heating, the selectivity will generally progress back to neutral, and the solvent will adopt a more uniform distribution. Accordingly, the phase behavior follows a diagonal trajectory back across the phase map toward the behavi or controlled by the block copolymer composition. A rich variety of thermotropic OOTs and ODTs are thus accessible. Central to these phenomena is the strong temperature dependence of the solvent selectivity toward the isoprene block. Work in progress is aimed at establishing a quantitative $\chi_{\text {PI-DBP/DEP }}$ parameter using a previously established SANS-SCMFT method. ${ }^{6,12}$

Several novel features emerge upon increasing the selectivity that are not captured by the trajectory approach. First, a relatively large window of coexisting $L$ and $C_{2}$ appears in DEP between the respective single phases, which is attributed to a destabilization of the $\mathrm{G}_{2}$ phase as a result of a heightened free energy penalty for packing frustration. Second, the spherical phase adopts a face-centered-cubic lattice in DEP and not the body-centered-cubic lattice found in the DBP and C14 solutions and neat block copolymers. The full explanation for this phenomenon is unresol ved. Third, a "reentrant" thermotropic ODT is observed for a $\phi=0.2$ solution near the lyotropic ODT, in which a disordered suspension of micelles swell, adopt a fcc lattice, and then disperse into polymer chains upon heating.

Finally, the concentration scaling of the domain period, $d^{*}$, depends strongly on the solvent selectivity. Addition of a highly selective solvent to the lamellar morphol ogy increases $d^{*}$ as the chains stretch to reduce their interfacial area. As the solvent becomes less selective with increasing temperature, the stretching becomes less severe and the concentration dependence weakens. Eventually, there is a crossover to $d^{*}$ decreasing with added solvent as the reduced segregation permits the chains to relax normal to the interface. In the high temperature limit the results agree with the previously found neutral solvent scaling of $d^{*} \sim \phi^{1 / 3}$. This phenomenon is captured by SCMF calculations 
with varying solvent selectivity.

Acknowledgment. This work was supported by the National Science Foundation through Awards DMR9528481 and DMR-9901087; the Center for Interfacial Engineering, an NSF-supported Engineering Research Center at the University of Minnesota; and a University of Minnesota Doctoral Dissertation Fellowship awarded to K.J.H. The assistance of Mary Beth Kossuth and Dr. Martin Vigild with the SAXS measurements is appreciated. We thank Bryant Pudil, Mary Beth Kossuth, and Dr. Damian Hajduk for helpful discussions.

\section{References and Notes}

(1) Hamley, I. W. The Physics of Block Copolymers; Oxford University Press: New York, 1998.

(2) Bates, F. S.; Fredrickson, G. H. Annu. Rev. Phys. Chem. 1990, $41,525$.

(3) Balsara, N. P.; Perahia, D.; Safinya, C. R.; Tirrell, M.; Lodge, T. P. Macromolecules 1992, 25, 3896.

(4) Hashimoto, T.; Shibayama, M.; Kawai, H. Macromolecules 1983, 16, 1093.

(5) Shibayama, M.; Hashimoto, T.; Hasegawa, H.; Kawai, H. Macromolecules 1983, 16, 1427.

(6) Lodge, T. P.; Hamersky, M. W.; Hanley, K. J .; Huang, C.-I. Macromol ecules 1997, 30, 6139.

(7) Lodge, T. P.; Pan, C.; J in, X.; Liu, Z.; Zhao, J .; Maurer, W. W.; Bates, F. S. J . Polym. Sci., Polym. Phys. Ed. 1995, 33, 2289.

(8) Takahashi, Y.; Kitade, S.; Noda, M.; Ochai, N.; Noda, I.; I mai, M.; Matsushita, Y. Polym. J . 1998, 30, 388.

(9) Hanley, K. J .; Lodge, T. P. J . Polym. Sci., Polym. Phys. Ed. 1998, 36, 3101.

(10) Sakamoto, N.; Hashimoto, T.; Han, C. D.; Kim, D.; Vaidya, N. Y. Macromolecules 1996, 30, 5321.

(11) Sakurai, S.; Hashimoto, T.; Fetters, L. J . Macromolecules 1996, 29, 740.

(12) Huang, C.-I.; Chapman, B. R.; Lodge, T. P.; Balsara, N. P. Macrmolecules 1998, 31, 9384.

(13) Banaszak, M.; Whitmore, M. D. Macromolecules 1992, 25, 3406.

(14) Whitmore, M. D.; Noolandi, J .J . Chem. Phys. 1990, 93, 2946

(15) Whitmore, M. D.; Vavasour, J. D. Macromol ecules 1992, 25 , 2041.

(16) Huang, C.-I.; Lodge, T. P. Macromolecules 1998, 31, 3556.

(17) Olvera de la Cruz, M. I. Chem. Phys. 1989, 90, 1995.

(18) Fredrickson, G. H.; Leibler, L. Macromol ecules 1989, 22, 1238.

(19) Hong, K. M.; Noolandi, J. Macromol ecules 1983, 16, 1083.

(20) Helfand, E.; Tagami, Y. J . Chem. Phys. 1972, 56, 3592.

(21) Tuzar, Z.; Kratochvil, P. Surface and Colloidal Science; Matijevic, E., Ed.; Plenum Press: New York, 1993; Vol. 15.

(22) Lodge, T. P.; Xu, X.; Ryu, C. Y.; Hamley, I. W.; Fairclough, J. P. A.; Ryan, A. J .; Pedersen, J . S. Macromol ecules 1996, 29, 5955.

(23) Hamley, I. W.; Fairclough, J . P. A.; Ryan, A. J .; Ryu, C. Y.; Lodge, T. P.; Gleeson, A. J .; Pedersen, J . S. Macromolecules 1998, 31, 1188.
(24) Shibayama, M.; Hashimoto, T.; Kawai, H. Macromolecules 1983, 16, 16

(25) Hashimoto, T.; Shibayama, M.; Kawai, H. Macromolecules 1983, 16, 361.

(26) McConnell, G. A.; Gast, A. P.; Huang, J . S.; Smith, S. D. Phys. Rev. Lett. 1993, 71, 2102.

(27) McConnell, G. A.; Gast, A. P. Macromolecules 1997, 30, 435.

(28) Hajduk, D. A.; Kossuth, M. B.; Hillmyer, M. A.; Bates, F. S. J. Phys. Chem. B 1998, 102, 4269.

(29) Alexandridis, P.; Olsson, U.; Lindman, B. Langmuir 1998 14, 2627.

(30) Mortensen, K.; Pedersen, J. S. Macromolecules 1993, 26, 807

(31) Almgren, M.; Brown, W.; Hvidt, S. Colloid Polym. Sci. 1995, $273,2$.

(32) Alexandridis, P.; Zhou, D.; Khan, A. Langmuir 1996, 12, 2690.

(33) Mortensen, K.; Brown, W.; J orgensen, E. Macromol ecules 1994, 27, 5654

(34) Mortensen, K. Europhys. Lett. 1992, 19, 599.

(35) Wanka, G.; H offmann, H.; Ulbricht, W. Macromol ecules 1994 27,4145

(36) Lodge, T. P.; Fredrickson, G. H. Macromolecules 1992, 25, 5643.

(37) Garetz, B. A.; Newstein, M. C.; Dai, H. J .; J onnalagadda, S V.: Balsara, N. P. Macromolecules 1993, 26, 3151.

(38) Hajduk, D.; Takenouchi, H.; Hillmyer, M. A.; Bates, F. S.; Vigild, M. E.; Almdal, K. Macromolecules 1997, 30, 3788.

(39) Hajduk, D. A.; Harper, P. E.; Gruner, S. M.; Honeker, C. C. Kim, G.; Thomas, E. L.; Fetters, L. J . Macromolecules 1994, 27, 4063.

(40) Förster, S.; Khandpur, A. K.; Zhao, J .; Bates, F. S.; Hamley, I. W.; Ryan, A. J .; Bras, W. Macromol ecules 1994, 27, 6922.

(41) Villacampa, M.; Diaz de Apodaca, E.; Quintana, J . R.; Katime, I. Macromol ecules 1995, 28, 4144.

(42) Zhou, Z.; Chu, B.; Peiffer, D. G. J . Polym. Sci., Polym. Phys. Ed. 1994, 32, 2135

(43) Xu, R.; Winnik, M. A.; Hallett, F. R.; Riess, G.; Croucher, M. D. Macromolecules 1991, 24, 87.

(44) Qin, A.; Tian, M.; Ramireddy, C.; Webber, S. E.; Munk, P.; Tuzar, Z. Macromolecules 1994, 27, 120.

(45) Khandpur, A. K.; Förster, S.; Bates, F. S.; Hamley, I. W. Ryan, A. J .; Bras, W.; Almdal, K.; Mortensen, K. Macromolecules 1995, 28, 8796.

(46) Ryu, C. Y.; Lodge, T. P. Macromolecules 1999, 32, 7190.

(47) Matsen, M. W. Macromolecules 1995, 28, 5765.

(48) Matsen, M. W.; Bates, F. S. J . Chem. Phys. 1997, 106, 2436.

(49) Matsen, M. W.; Bates, F. S. Macromol ecules 1996, 29, 7641

(50) Matsen, M. W.; Bates, F. S. Macromolecules 1996, 29, 1091.

(51) Matsen, M. W.; Bates, F. S. J . Polym. Sci., Polym. Phys. Ed. 1997, 35, 945

(52) Sadoc, J . F.; Charvolin, J . J . Phys. (Paris) 1986, 47, 683.

(53) Gruner, S. M. J . Phys. Chem. B 1989, 93, 7562.

(54) Hamley, I. W.; Pople, J . A.; Diat, O. Colloid Polym. Sci. 1998 $276,446$.

(55) Pople, J. A.; Hamley, I. W.; Fairclough, J . P. A.; Ryan, A.J .; Komanschek, B. U.; Gleeson, A. J .; Yu, G.-E.; Booth, C. J Macromolecules 1997, 30, 5721.

(56) Deng, N.-J .; Luo, Y.-Z.; Tanodekaew, S.; Bingham, N.; Attwood, D.; Booth, C. J . Polym. Sci., Polym. Phys. Ed. 1995, 33, 1085.

MA000318B 